\title{
Optimal Power Management in Wireless Control Systems
}

\author{
Konstantinos Gatsis, Student Member, IEEE, Alejandro Ribeiro, Member, IEEE, \\ and George J. Pappas, Fellow, IEEE
}

\begin{abstract}
This paper considers the control of a linear plant when plant state information is being transmitted from a sensor to the controller over a wireless fading channel. The power allocated to these transmissions determines the probability of successful packet reception and is allowed to adapt online to both channel conditions and plant state. The goal is to design plant input and transmit power policies that minimize an infinite horizon cost combining power expenses and the conventional linear quadratic regulator control cost. Since plant inputs and transmit powers are in general coupled, a restricted information structure is imposed allowing them to be designed separately. Under this information structure the standard LQR controller becomes the optimal plant input policy, while the optimal communication policy follows a Markov decision process minimizing transmit power at the sensor and state estimation error at the controller. The optimal power adaptation to channel and plant states is examined qualitatively for general forward error correcting codes. In the particular case of capacity achieving codes event-triggered policies are recovered, where the sensor decides whether to transmit or not based on plant and channel conditions. Approximate dynamic programming is employed to derive a family of tractable suboptimal communication policies exhibiting the same qualitative features as the optimal one. The performance of our suboptimal policies is shown in simulations and is contrasted to other simple transmission policies.
\end{abstract}

Index Terms-Networked control systems, wireless fading channels, power adaptation, linear quadratic control, control/communication separation, event-triggered design.

\section{INTRODUCTION}

The networked control systems studied in this paper are characterized by the separation of sensing and actuation in different physical devices with control loops involving the communication of plant state information over a wireless channel. When sensor and controller communicate over a wireless channel the cost of controlling the plant gets mixed with the cost of sending plant state information from the sensor to the controller. The more information the sensor conveys the more precise actuation becomes, but the resulting increase in power consumption at the sensor leads to rapid depletion of its energy resources. It is therefore apparent that a tradeoff emerges between plant performance and power consumption. To quantify this tradeoff we study the problem of selecting

Work in this paper is supported in part by NSF CNS-0931239, and by the TerraSwarm Research Center, one of six centers supported by the STARnet phase of the Focus Center Research Program (FCRP) a Semiconductor Research Corporation program sponsored by MARCO and DARPA. Preliminary versions of this work can be found in [1].

The authors are with the Department of Electrical and Systems Engineering, University of Pennsylvania, 200 South 33rd Street, Philadelphia, PA 19104. Email: \{kgatsis, aribeiro, pappasg\}@ seas.upenn.edu. plant inputs and power management policies that minimize a joint cost that accounts for the plant regulation cost and the cost of conveying information from the sensor to the controller.

\section{A. Related literature}

Early works on networked control systems ignore the cost of conveying information and focus their analysis on the performance of control loops when various communication effects are taken into account. For packet-based communication over analog erasure channels, necessary and sufficient requirements for stability are analyzed, and controllers and estimators are designed to counteract random packet drops and delays in, e.g., [2]-[5] and references therein. Alternative models of analog channels as input-output systems with channel randomness treated as stochastic model uncertainty have been examined [6], facilitating controller synthesis using robust control techniques [7]. A different set of issues arise when loops close over digital channels with data-rate constraints. In such case quantization effects become important and apart from the controller design an efficient encoding/decoding scheme is required [8]. Fundamental limits like the minimum bit rate for stabilization are also known; see, e.g., [9].

For networked control architectures where communication is not treated as a limitation but becomes an active part of the design, the setup typically departs from the classic periodic communication paradigm. This leads to frameworks such as event-triggered sampling [10], [11] and control [12], [13], or self-triggered control [14]. The underlying concept in these contributions is to prolong the time elapsed between successive sampling or input updates as long as some Lyapunov-like plant performance criterion is satisfied. Such schemes exhibit in general an average communication/update rate lower than periodic schemes that attain similar plant performance. However, communication costs are not explicitly accounted for in the triggering design.

Communication costs are explicitly modeled in the context of remote state estimation in [15]-[18]. In this framework a sensor measuring the plant state decides whether to transmit its value to an estimator or not and each transmission incurs a fixed cost. The overall goal is to minimize the estimation error cost and the communication penalties aggregated over time. The optimal communication is event-triggered [15], similar to, e.g., [12], [13], meaning that transmissions are triggered when the estimation error exceeds a threshold. Computing the optimal transmission-triggering sets is not tractable, motivating the development of suboptimal schemes [17], [18]. Related 
contributions consider plant and communication controllers jointly optimal with respect to linear quadratic and communication cost assuming again a fixed cost per transmission [19], [20]. The problem is more complex than the case of simple state estimation but a separation principle can be imposed [20], and optimal inputs and schedules can be found by dynamic programming for a finite horizon. The characterization and determination of jointly optimal plant and transmission policies in this context is otherwise open. We note however that in the case of control over digital channels, aspects of jointly optimal encoder and controller design have recently been studied [21].

\section{B. Contributions and summary}

Instead of accounting for communication cost in terms of transmissions, in this paper we are interested in the allocation of the actual resource used by the wireless sensors to communicate, namely, transmitted power. This perspective permits the incorporation of fading effects in the wireless channel. Fading refers to large unpredictable variations in wireless channel transferences whose mitigation involves extensive use of power adaptation to channel conditions [22, Ch. 3,4]. Besides counteracting fading effects power adaptation may be also helpful in closed-loop control to protect some transmissions more than others, e.g., to increase the likelihood of successful packet decoding when the plant state deviates from target. In this paper transmit power is allowed to adapt to both, the random fading channel state and the plant state. The allocated power and the fading channel state determine the likelihood of successful packet decoding at the receiver by a known complementary error function (Section II). This communication model has been used in the context of remote state estimation [23], [24] and can be regarded as a generalization of the erasure channel with i.i.d. dropouts of, e.g., [2]-[5], since here the probability of packet drops is actively controlled by an online transmit power policy. We note that an alternative model where transmit power affects directly the value of the received analog message, instead of the packet decoding, is examined in [25, Ch. 7] but does not consider adaptation to fading effects.

Given our communication model we are interested in the trade-off between closed-loop plant performance and power resources. To this end we combine the transmit power with a conventional linear quadratic regulator (LQR) cost to form an aggregate infinite horizon cost that we seek to minimize through proper joint selection of plant and power control policies (Section II-A). For the novel problem formulation proposed we begin by identifying restricted information structures that permit decoupling of plant input and power control policies (Section II-B). For this particular information structure the usual LQR control law becomes optimal at the controller side while the optimal communication policy at the transmitter follows from a Markov decision process (MDP) formulation accounting for transmit power and the state estimation error at the controller (Section III). The optimal power control policy is then expressed in terms of a value function solving the MDP problem (Section IV). While this does not allow computation of optimal policies it does allow us to understand the qualitative characteristics of the optimal resource allocation.
In contrast to the work in, e.g., [15]-[17], [19] where transmission is based just on plant state, the availability of channel state information at the transmitter leads to new insights on the optimal communication policy. In particular when channel gain is low or estimation error small no transmission is triggered, since it would be costly or unnecessary respectively. On the other hand, similar to the above work, there is an event/set of plant and channel states where transmission is triggered, but the optimal power allocation on this event still needs to adapt to the channel and plant states. Alternatively, our power management policy can be viewed as a 'soft' version of the event-triggered paradigm of, e.g., [12], as instead of just deciding whether to transmit or not we select how much power to allocate to the transmission attempt. This interpretation is further fostered by the realization that conventional eventtriggered policies emerge as the optimal communication when the sensor uses capacity achieving forward error correcting codes (Section IV-A).

Finally since optimal communication is not computationally tractable we devise suboptimal power control policies using approximate dynamic programming, in particular rollout algorithms (Section V). These policies maintain the same qualitative characteristics as the optimal policies for general error correcting or capacity achieving codes, and this is verified in numerical simulations (Section VI). Our rollout policies are shown to have significant performance benefits compared to other simple policies that adapt only to channel conditions and not the plant state, such as the ones proposed in [23], [24]. We close the paper with conclusions and suggestions on how the adopted model can be used for more complex wireless communication/control network design problems in future work (Section VII).

Notation: Let $\mathcal{N}_{\mu, \Sigma}$ denote the $n$-dimensional Gaussian distribution with mean $\mu$ and covariance $\Sigma$. For a square matrix $M \in \mathbb{R}^{n \times n}$ let $\lambda_{\max }(M), \lambda_{\min }(M)$ denote respectively the largest and smallest eigenvalues in magnitude. For compactness a set of variables $\left\{x_{k}, x_{k+1}, \ldots, x_{k+t}\right\}$ is denoted by $x_{k: k+t}$. Subscripts of variables as in $x_{k}, x_{k+1}$ denote discrete time. When time index $k$ is clear from the context, subscripts are omitted and the respective variables are denoted as $x, x_{+}$.

\section{Problem Formulation}

We consider the architecture shown in Fig. 1 deployed to control a discrete-time linear time-invariant plant described by the difference equation

$$
x_{k+1}=A x_{k}+B u_{k}+w_{k}, k \geq 0,
$$

where $x_{k} \in \mathbb{R}^{n}$ is the plant's state with $x_{0}$ given, $u_{k} \in \mathbb{R}^{m}$ the driving input, and $\left\{w_{k}, k \geq 0\right\}$ is the process noise composed of independent identically distributed (i.i.d) $n$-dimensional Gaussian random variables $w_{k} \sim \mathcal{N}_{0, W}$ with zero mean and covariance $W$. We assume the plant is unstable $\left(\lambda_{\max }(A)>1\right)$ but that $(A, B)$ is stabilizable.

The wireless control system considered in this paper includes a sensor/transmitter collecting state measurements $x_{k}$ that it communicates with power $p_{k} \in\left[0, p_{\max }\right]$ over a wireless fading channel with coefficient $h_{k}$. At the other side of the 


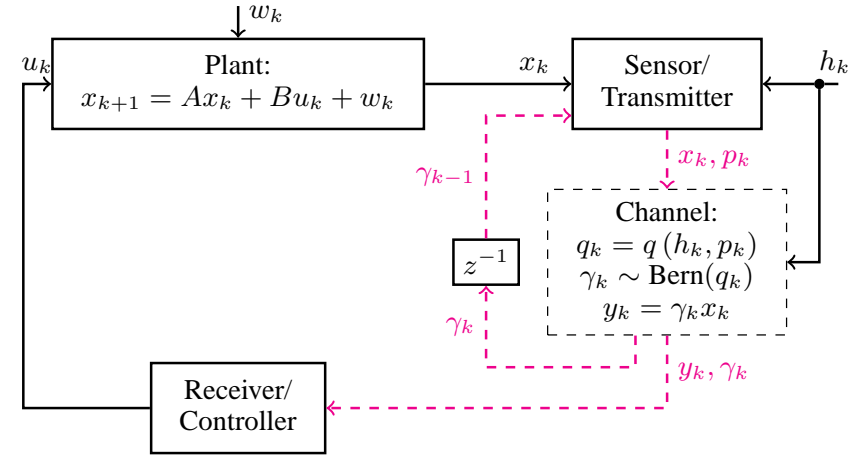

Fig. 1. Wireless control system architecture. A sensor measures the plant and wireless fading channel states $x_{k}, h_{k}$ respectively and transmits with power $p_{k}$. Messages are successfully decoded at the controller with probability $q_{k}$ that depends on the channel state $h_{k}$ and the power $p_{k}$. The sensor receives acknowledgments with a one-step delay.

channel the receiver/controller uses the received information to determine a control input $u_{k}$ that it feedbacks into the plant. The effects of state quantization and transmission delays are considered negligible and are thus ignored henceforth.

Due to propagation effects the channel gain $h_{k}$ changes unpredictably [22, Ch. 3]. We adopt the standard block fading model of wireless communications whereby channels $\left\{h_{k}, k \geq 0\right\}$ are modeled as i.i.d. random variables taking values in the positive reals $\mathbb{R}_{+}$according to some known distribution $m_{H}$ and are independent of the plant process noise $\left\{w_{k}, k \geq 0\right\}$. We make the technical assumption that the distribution $m_{H}$ of the channel state has a probability density function on $\mathbb{R}_{+}$. To allow for transmissions adapted to the current channel conditions the transmitter has access to the channel state information $h_{k}$ before transmitting at time $k-$ the development is equally valid if estimates are available in lieu of $h_{k}$ as discussed in Remark 2.

At the controller side the received signal includes the information bearing signal and additive white Gaussian noise (AWGN). The noise power is denoted by $N_{0}$ and the power of the information bearing signal is the product $h_{k} p_{k}$. Assuming the receiver also has channel state information, successful decoding of the transmitted packet is determined by the signal to noise ratio (SNR) at the receiver defined as $\mathrm{SNR}_{k}:=$ $h_{k} p_{k} / N_{0}$. More precisely, given the particular type of modulation and forward error correcting (FEC) code used, the SNR determines the probability of successful detection $q_{k}$. To keep the analysis general we define a generic complementary error function

$$
q_{k}=q\left(h_{k}, p_{k}\right),
$$

mapping $\mathrm{SNR}_{k}:=h_{k} p_{k} / N_{0}$ to the probability $q_{k}$. We assume that $q(h, p)$ is a known increasing function of the product $h p$ - see Remark 1.

Considering packet decoding as a part of the communication process, we can model communication as a sequence of indicator variables $\gamma_{k}$ taking value $\gamma_{k}=1$ when information is successfully decoded and $\gamma_{k}=0$ otherwise. Variables $\gamma_{k} \sim \operatorname{Bern}\left(q_{k}\right)$ are Bernoulli distributed with time-varying success probabilities $q_{k}$ given by (2). With this communication model the controller receives the output of the decoding

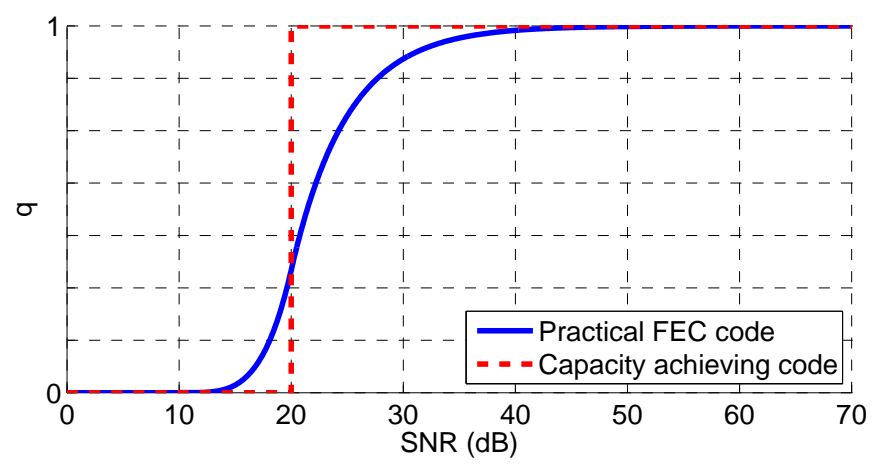

Fig. 2. Complementary error function for FEC and capacity achieving codes. The probability of successful decoding $q$ for a practical FEC code is a sigmoid function of the received SNR $=h p / N_{0}$, while for a capacity achieving code a threshold value $\mathrm{SNR}_{0}$ determines whether a packet is successfully received.

process which we model by the signal $y_{k}=\gamma_{k} x_{k}$. We further assume that the controller also gets $\gamma_{k}$ so that it can distinguish between the cases $x_{k}=0$ and $\gamma_{k}=0$. Packet receipt acknowledgment $\gamma_{k}$ is also sent to the sensor as provided by 802.11 and TCP protocols. We assume lossless acknowledgments, so that the sensor knows what information is received at the controller.

The problem addressed in this paper is the joint design of the control inputs $u_{k}$ and the transmit powers $p_{k}$. The control input $u_{k}$ is determined by the received information $y_{0: k}, \gamma_{0: k}$. The power $p_{k}$ is determined as a function of the plant state measurements $x_{0: k}$, the observed channel realizations $h_{0: k}$, and the controller acknowledgments $\gamma_{0: k}$. Informally, to conserve power at the sensor side we want to transmit information only when the state $x_{k}$ deviates from its desired value or when the channel realization $h_{k}$ is favorable. In the first case transmission is necessary to keep the plant under control. In the latter case the transmission cost is minimal. A formal problem specification is presented in the next section after the following remarks.

Remark 1. The error profiles $1-q\left(h_{k}, p_{k}\right)$ of particular FEC codes are difficult to determine analytically but can be measured in actual or simulated experiments [26], [27]. Typically $q\left(h_{k}, p_{k}\right)$ is a sigmoid function of $h_{k} p_{k}$ with exponential tails as depicted in Fig. 2. In the theoretical limit, correct decoding depends on the channel capacity $C_{k}=\mathcal{W} \log _{2}\left(1+\mathrm{SNR}_{k}\right)$, where $\mathcal{W}$ is the channel bandwidth. If the packet is transmitted at a rate smaller than $C_{k}$ bits per second it is almost surely successfully decoded, and it is almost surely incorrectly decoded otherwise. Thus, we can write the successful decoding probability as the indicator function

$$
q\left(h_{k}, p_{k}\right)=\mathbb{I}\left(\frac{h_{k} p_{k}}{N_{0}} \geq \mathrm{SNR}_{0}\right),
$$

for some constant $\mathrm{SNR}_{0}$. Determining the threshold $\mathrm{SNR}_{0}$ requires specification of the sampling rate and quantization resolution of the state $x_{k}$. With $\alpha$ samples per second and $\beta$ bits per sample we require a transmission rate of $\alpha \beta$ bits per second. The SNR threshold is then given by $\mathrm{SNR}_{0}=$ $2^{\alpha \beta / \mathcal{W}}-1$. Our interest in (3) is conceptual as it will allow us to recover results in event-triggered communication [15] as 


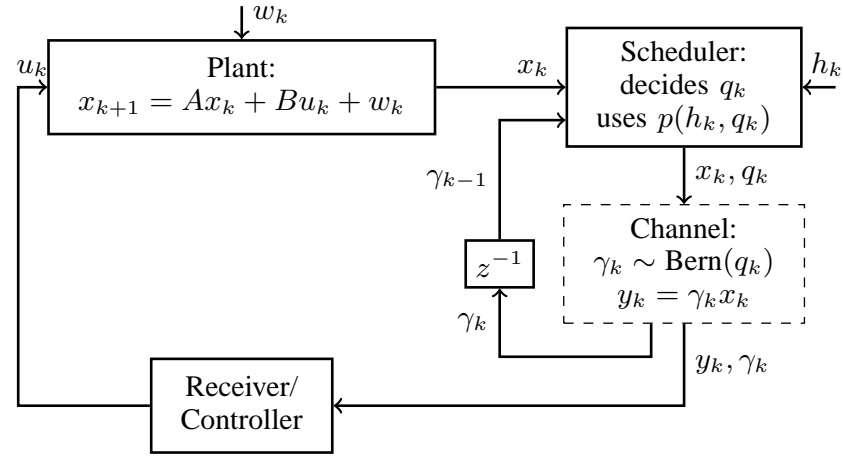

Fig. 3. Equivalent wireless control system architecture. A scheduler decides the successful decoding probability $q_{k}$ and transmits the state measurement $x_{k}$ with the required power $p_{k}=p\left(h_{k}, q_{k}\right)$. The controller receives the message with probability $q_{k}$.

arising from the use of capacity achieving codes - see Section IV-A. The form of (3) is shown in Fig. 2.

Remark 2. The assumption that channel state information (CSI) is available at the transmitter is typical in modern wireless communication setups [22, Ch. 9]. To measure the wireless channel conditions a short pilot signal of fixed power can be sent from the transmitter and then the fading characteristics can be estimated at the receiver and sent back to the transmitter by utilizing the reverse channel. Although accurate CSI is difficult to acquire at the transmitter side, our development is still valid if channel estimates are available in lieu of the actual channel value $h_{k}$. Reinterpreting $h_{k}$ as an estimate of the fading coefficient the complementary error function $q(h, p)$ in (2) captures not only the success of decoding but also the uncertainty over the real channel gain. It suffices to integrate $q(h, p)$ with respect to the conditional distribution of the channel realization given the estimate.

Remark 3. There is a distinction to be made between errors that are detected by the receiver and errors that are undetected and may confuse the controller. The model here handles the former and ignores the latter. This is justified because practical communication schemes include the use of cyclic redundancy checks (CRC) for error detection that can drive the probability of undetected errors to very small values [28, Ch. 4]. The use of simple CRCs reduces the probability of undetected errors to $10^{-3}$, while longer codes can reduce this probability to $10^{-7}$.

\section{A. Joint design of plant and power control}

To formulate the joint design of plant controller and power management we introduce an equivalent architecture. In view of (2), choosing $p_{k}$ is equivalent to choosing the desired probability of successful decoding $q_{k}$ at time $k$ and transmitting with the minimum required power to achieve this $q_{k}$, namely

$$
p_{k}=p\left(h_{k}, q_{k}\right):=\inf \left\{0 \leq p \leq p_{\max }: q\left(h_{k}, p\right) \geq q_{k}\right\} .
$$

We can therefore interpret $q_{k}$ as our decision variable with $p\left(h_{k}, q_{k}\right)$ denoting the cost of selecting transmission success probability $q_{k}$. This leads to the equivalent control system architecture shown in Fig. 3 where a scheduler block responsible for deciding $q_{k}$ replaces the sensor/transmitter block of Fig 1. Our formulation generalizes the simple transmit-or-not decision as considered in, e.g., [15].

We note for future reference that the assumed monotonicity of the function $q(h, p)$ on the product $h p$ implies that the power function $p(h, q)$ is increasing in $q$ and decreasing in $h$. Using maximum power $p_{\max }$, the transmitter can achieve a maximum successful decoding probability $q_{\max }(h):=$ $q\left(h, p_{\max }\right)$ for a given channel state $h$. Therefore, the decision variables $q_{k}$ belong in the interval $\left[0, q_{\max }\left(h_{k}\right)\right]$. We also make the following assumptions.

Assumption 1. The maximum achievable successful decoding probability $q_{\max }(h)$ satisfies

$$
\mathbb{E}_{h} q_{\max }(h)>q_{\text {crit }}:=1-1 / \lambda_{\max }(A)^{2},
$$

where expectation is taken over the channel distribution $m_{H}$.

Assumption 2. For any channel realization $h$, the function $p(h, q)$ in (4) is continuous in the successful decoding probability variable $q$.

Assumption 1 is essentially a stability condition, which as we will see in the following section states that transmitter has enough power to keep the plant state bounded in second moment, and it will be used to establish our main Theorems 1 and 2. Assumption 2 is of a technical nature and will be used in Theorem 2.

In the architecture of Fig. 3 the communication decision $q_{k}$ is chosen as a function of the information available at the sensor, while the plant control signal $u_{k}$ is a function of the information available at the controller. These choices are in general allowed to be randomized. The sequence $\pi:=$ $\left\{q_{0}, q_{1}, \ldots\right\}$, or equivalently the power allocation $\left\{p_{0}, p_{1}, \ldots\right\}$, is termed the communication policy, whereas the sequence $\theta:=\left\{u_{0}, u_{1}, \ldots\right\}$ denotes the control policy. With fixed policies $\pi, \theta$, all random variables are defined on an appropriate product space and have a measure that we denote as $\mathbb{P}^{\pi, \theta}$. We use $\mathbb{E}^{\pi, \theta}$ to signify integration with respect to $\mathbb{P}^{\pi, \theta}$, which we simplify to $\mathbb{E}$ when not leading to confusion. We remark that sensor and controller know each other's policy.

The policy pair $(\pi, \theta)$ incurs a control cost and a communication cost. As a control cost we adopt the standard linear quadratic regulator cost

$$
J_{\mathrm{LQR}}^{N}(\pi, \theta):=\mathbb{E}^{\pi, \theta} \sum_{k=0}^{N-1} x_{k}^{T} Q x_{k}+u_{k}^{T} R u_{k},
$$

for some pair of matrices $R>0$ and $Q \geq 0$, with $\left(A, Q^{1 / 2}\right)$ detectable. The communication cost is given by the expected power consumption

$$
J_{\mathrm{PWR}}^{N}(\pi, \theta):=\mathbb{E}^{\pi, \theta} \sum_{k=0}^{N-1} p\left(h_{k}, q_{k}\right) .
$$

To quantify the tradeoff between plant performance and power consumption we combine the LQR cost in (6) and the power cost in (7) into the limit aggregate cost

$$
J(\pi, \theta):=\limsup _{N \rightarrow \infty} 1 / N\left[J_{\mathrm{LQR}}^{N}(\pi, \theta)+\lambda J_{\mathrm{PWR}}^{N}(\pi, \theta)\right],
$$


for some positive constant $\lambda>0$. Our goal is to design plant and power control policies $\theta$ and $\pi$ respectively that minimize the joint cost (8). These policies depend on what information is available to the sensor and controller. The specific information structure considered in this paper is introduced next.

\section{B. Information structure}

Denote as $O_{k}$ the information known at the controller side at time $k$ just before deciding the input $u_{k}$. This information includes the given initial plant state $x_{0}$, the history of decoding success variables $\gamma_{0: k}$ and the decoded signals $y_{0: k}$, as well as the previously chosen control inputs $u_{0: k-1}$, i.e.,

$$
O_{k}:=\left\{x_{0}, \gamma_{0: k}, y_{0: k}, u_{0: k-1}\right\} \text {. }
$$

Then the control input $u_{k}$ is chosen as a function of $O_{k}$, or more formally, measurable with respect to the $\sigma$-field generated by $O_{k}$.

Given the possibility of lost packets as indicated by $\gamma_{k}=0$, the controller has partial information on the plant state $x_{k}$. It is then of importance to study the MMSE estimate $\mathbb{E}^{\pi, \theta}\left(x_{k} \mid O_{k}\right)$. This estimation is complicated by the fact that the event $\gamma_{k}=0$ possibly contains information about the state $x_{k}$ through the dependence of the probability $q_{k}$ on the value of $x_{k}$ - see Remark 4. To avoid this complication we discard the information given by events of the form $\gamma_{k}=0$. Formally, define $\tau_{k}:=\max \left\{0 \leq l \leq k: \gamma_{l}=1\right\}$ as the time of the last successful transmission by time $k$ and define the sequence

$$
G_{k}:=\left\{x_{0}, \gamma_{0: \tau_{k}}, y_{0: \tau_{k}}, u_{0: k-1}\right\}
$$

with $G_{0}=\left\{x_{0}\right\}$. When $\gamma_{k}=1, G_{k}$ coincides with $O_{k}$. When $\gamma_{k}=0, G_{k}$ only contains information received till the last successful transmission which occurred at time $\tau_{k}<k$.

We restrict attention to control policies $\theta$ selecting inputs $u_{k}$ as functions of $G_{k}$, possibly randomized, and denote the set of all such policies by $\Theta$. Unlike $\mathbb{E}^{\pi, \theta}\left(x_{k} \mid O_{k}\right)$, the state MMSE estimate $\hat{x}_{k}:=\mathbb{E}^{\pi, \theta}\left(x_{k} \mid G_{k}\right)$ with respect to $G_{k}$ is easy to compute. When $\gamma_{k}=1$ the state $x_{k}=y_{k}$ becomes known at the receiver side. When $\gamma_{k}=0$ no new information becomes available and $\hat{x}_{k}$ is obtained by propagating $\hat{x}_{k-1}$ through the plant's dynamics in (1). Put together, we get

$$
\hat{x}_{k}:=\mathbb{E}^{\pi, \theta}\left(x_{k} \mid G_{k}\right)=\left\{\begin{array}{ll}
y_{k} & \text { if } \gamma_{k}=1, \\
A \hat{x}_{k-1}+B u_{k-1} & \text { if } \gamma_{k}=0
\end{array},\right.
$$

with $\hat{x}_{0}=x_{0}$ since the initial state is given.

At the other side of the link at time $k$ the sensor/transmitter has access to the channel realization $h_{k}$ and the plant state $x_{k}$ which allows selection of the successful transmission probability $q_{k}$ to depend on the values of both of $h_{k}, x_{k}$. This affects the controller design however, because when the controller decides $u_{k-1}$ to control $x_{k}$, it should consider the indirect effect on $q_{k}$. This information structure renders the joint communication and control co-design problem hard to analyze. To overcome this, we restrict transmission policies to depend on the channel state $h_{k}$ and the information about plant state $x_{k}$ that the controller does not know. More precisely consider the difference between the sensor measurement $x_{k}$ and the controller's estimate $\hat{x}_{k}$ by (11) if the $k$ th packet is not successfully decoded, that is

$$
\varepsilon_{k}:=x_{k}-\left(A \hat{x}_{k-1}+B u_{k-1}\right),
$$

with $\varepsilon_{0}:=0$. Observe that the term in the parenthesis is known to the sensor since by the acknowledgment mechanism the controller's previous estimate $\hat{x}_{k-1}$ and input $u_{k-1}$ can be replicated at the sensor. Alternatively the terms $\varepsilon_{k}$ can be viewed as the innovations of the controller's estimate (11) when a new message is received.

We restrict then information at the sensor side to the set $F_{k}$ defined as a collection of the channel history $h_{0: k}$, the history of innovations $\varepsilon_{0: k}$, and past decisions $q_{0: k-1}$, i.e.,

$$
F_{k}:=\left\{\varepsilon_{0: k}, h_{0: k}, q_{0: k-1}\right\} .
$$

We also add a technical requirement that the sensor selects maximum transmit power $p_{\max }$ when the innovation $\varepsilon_{k}$ gets too large, $\left\|\varepsilon_{k}\right\| \geq L$ for some positive constant $L>0$, and the channel gain $h_{k}$ is favorable, $h_{k} \geq h_{t}$ for some threshold $h_{t}>0$ on channel values. We assume that a positive success probability $q_{\max }\left(h_{t}\right)>0$ is achieved at this threshold and also that

$$
\int_{h \geq h_{t}} q_{\max }(h) d m_{H}(h)>q_{c r i t},
$$

where $q_{\text {crit }}$ is given in (5) and the integration is over the channel distribution $m_{H}$. Such a channel threshold exists by Assumption 1. We consider then communication policies $\pi$ selecting decoding success $q_{k}$ as functions of $F_{k}$ for each $k$, possibly randomized, satisfying $q_{k} \in Q\left(\varepsilon_{k}, h_{k}\right)$ where

$$
Q(\varepsilon, h):=\left\{\begin{array}{ll}
q_{\max }(h) & \text { if }\|\varepsilon\| \geq L \text { and } h \geq h_{t} \\
{\left[0, q_{\max }(h)\right]} & \text { otherwise }
\end{array} .\right.
$$

We denote the set of all such policies with $\Pi$. The technical power saturation requirement is inconsequential as we may pick $L$ arbitrarily large, and will be used to prove Proposition 2 and Theorem 2 in the sequel. Similar requirements have been introduced in [15], [16], however our setup is further complicated by the availability of the random channel states.

The proposed information structure is depicted in Fig. 4. The sensor block is split into a pre-processor and a scheduler. The pre-processor computes $\varepsilon_{k}$ based on the sample $x_{k}$ and the acknowledgment $\gamma_{k-1}$ and feeds it to the scheduler who, upon measuring the channel $h_{k}$ decides the transmission success probability $q_{k}$ while incurring power cost $p\left(h_{k}, q_{k}\right)$. Our goal in this paper is to study policies $\pi \in \Pi$ and $\theta \in \Theta$ that are optimal with respect to the joint objective (8), that is

$$
\underset{\pi \in \Pi, \theta \in \Theta}{\operatorname{minimize}} J(\pi, \theta) .
$$

In particular, the next section shows that the information structure we introduced allows optimal communication and control policies to be designed separately. The standard LQR controller is shown to be optimal and we then leverage this result to study optimal communication policies in Section IV and to develop tractable suboptimal policies in Section V.

Remark 4. If the controller uses the complete information $O_{k}$ to estimate $x_{k}$, the optimal plant estimate is not $\hat{x}_{k}$ as 


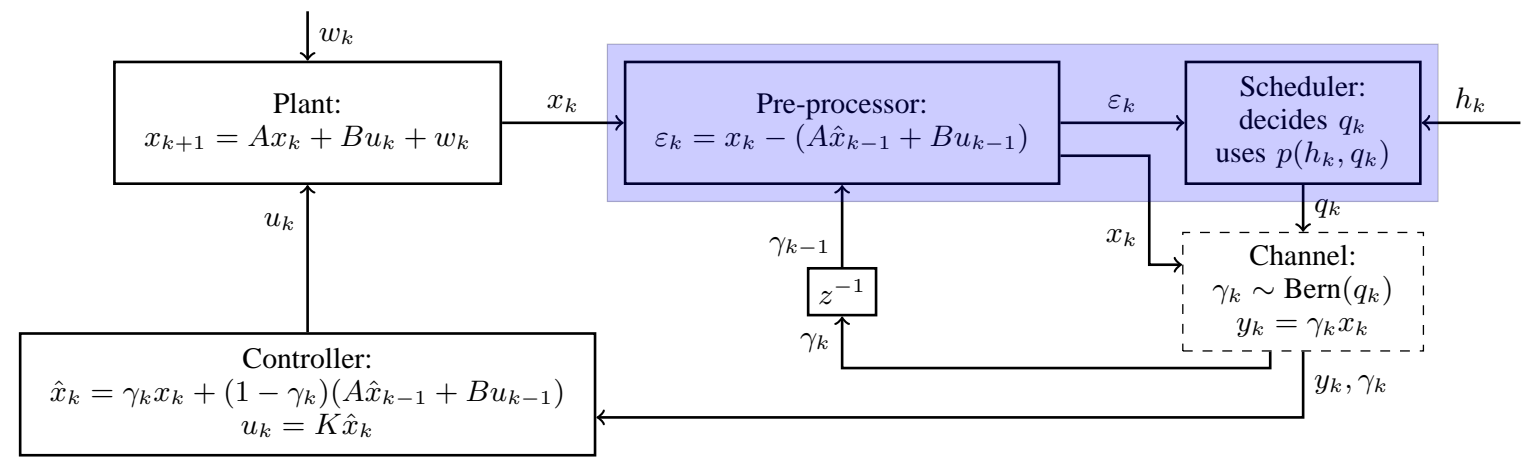

Fig. 4. Wireless control system with a restricted information structure. The sensor consists of two blocks. A pre-processor computes the error $\varepsilon_{k}$ given the measurement $x_{k}$ and the acknowledgment $\gamma_{k-1}$. A scheduler decides $q_{k}$ based on $\varepsilon_{k}$ and the channel state $h_{k}$, and transmits $x_{k}$ with the required power $p_{k}=p\left(h_{k}, q_{k}\right)$. The controller receives the message with probability $q_{k}$, computes the state estimate $\hat{x}_{k}$ and provides input $u_{k}$ to the plant.

given by (11). When a packet drop $\gamma_{k}=0$ is observed, and since the communication policy is known, the controller should consider the possibility that the sensor did not transmit at all, which could in general give indirect information about the value of $x_{k}$ - see also [19], [20] for a further discussion. The restriction to $G_{k}$ in (10) allows to overcome this issue and obtain linear dynamics of the estimation error $e_{k}$ and the related $\varepsilon_{k}$ as described next in (17),(18), but it is not needed for the separation result of Prop. 1 in the following section to hold.

\section{SEPARATION OF DESIGNS}

In this section we show that with the imposed restrictions on the information available at sensor and controller the control law $\theta \in \Theta$ and the communication policy $\pi \in \Pi$ can be designed separately. In particular the control policy has no effect on the estimation process at the receiver and by utilizing a separation principle the optimal controller becomes the standard linear quadratic one.

Let us denote the difference between the plant state and the estimate kept at the controller by $e_{k}:=x_{k}-\hat{x}_{k}$ and its covariance as seen at the controller by $\Sigma_{k}:=\mathbb{E}^{\pi}\left[e_{k} e_{k}^{T} \mid G_{k}\right]$. The estimation error dynamics can be found by subtracting (11) from the system dynamics (1) to get

$$
e_{k}=\left(1-\gamma_{k}\right)\left(A e_{k-1}+w_{k-1}\right),
$$

with $e_{0}=0$ since $x_{0}$ is given. Stabilizability of estimation error is guaranteed by Assumption 1. Indeed if transmitter were to use maximum power all the time the dynamics in (17) become a jump linear system since $\gamma_{k}$ are Bernoulli with constant probability equal to the left hand side of (5). Then condition (5) is sufficient for bounded second moment as, e.g., in [2, Theorem 2]. It is also tight in the sense that estimation error becomes unstable if $\mathbb{E}_{h} q_{\max }(h)<q_{\text {crit }}$.

Turning our attention to the innovation substituting $x_{k}$ by (1) in the definition of $\varepsilon_{k}$ in (12) gives $\varepsilon_{k}=A e_{k-1}+w_{k-1}$. The term $e_{k-1}$ equals $\left(1-\gamma_{k-1}\right) \varepsilon_{k-1}$ as seen by (17), therefore $\varepsilon_{k}$ evolves according to

$$
\varepsilon_{k}=\left(1-\gamma_{k-1}\right) A \varepsilon_{k-1}+w_{k-1},
$$

with initial value $\varepsilon_{0}=0$. The following proposition establishes a separation principle in our restricted information structure setup, stating that the control action has no effect on the quality of the future estimates at the controller.

Proposition 1. Consider any communication policy $\pi$ selecting successful decoding probabilities $q_{k}$ as functions of $F_{k}$ given in (13), possible randomized, with $\varepsilon_{k}$ defined in (12) and channel states $h_{k}$ independently drawn from a distribution $m_{H}$. Then at any step $k$ the distributions of the future processes $\left\{\varepsilon_{\ell}, q_{\ell}, \gamma_{\ell}, e_{\ell}, \ell>k\right\}$ given $G_{k}$ do not depend on the chosen control policy $\theta \in \Theta$.

Proof: First note that the processes $\left\{w_{k}, h_{k}, k \geq 0\right\}$ are by assumption independent of any other process. Then we follow an induction argument to prove the claim. At $k=0$, $\varepsilon_{0}$ is equal to $0, q_{0}$ depends only on $h_{0}$ and $\varepsilon_{0}, \gamma_{0}$ is an independent Bernoulli with success $q_{0}$, and $e_{0}$ is also 0 since $x_{0}$ is initially known. Consider then a time $k$ with a given $G_{k}$, the corresponding estimation error $e_{k}$ given $G_{k}$ having zero mean and covariance $\Sigma_{k}$, and a control input $u_{k}$ that is a function of $G_{k}$ as described by the control policy $\theta$. The term $\varepsilon_{k+1}$ equals $A e_{k}+w_{k}$, as indicated by the arguments preceding (18), which given $G_{k}$ has mean 0 and covariance $A \Sigma_{k} A^{T}+W$. The choice $q_{k+1} \in F_{k+1}$ by construction depends on past variables in $F_{k}$ which by causality do not depend on the action $u_{k}$, as well as the new variables $\varepsilon_{k+1}, h_{k+1}$ which are also independent of $u_{k}$. Also the distribution of $\gamma_{k+1} \sim \operatorname{Bern}\left(q_{k+1}\right)$ only depends on the distribution of $q_{k+1}$, and the same holds for $e_{k+1}$ which equals $\left(1-\gamma_{k+1}\right) \varepsilon_{k+1}$ again by the arguments preceding (18). To sum up all variables $\varepsilon_{k+1}, q_{k+1}, \gamma_{k+1}, e_{k+1}$ given $G_{k}$ do not depend on $u_{k}$.

The intuition behind this proposition is that the effect of control inputs is subtracted from $x_{k}$ when forming the innovation terms $\varepsilon_{k}$ in (12) that are fed to the communication policy $\pi$. Similar separation results based on innovation terms have been utilized in other communication/control design problems [9], [19], [20]. The above proposition restates the separation principle for our power allocation problem under channel state information.

Since the power cost $J_{\mathrm{PWR}}^{N}(\pi, \theta)$ in (7) only depends on pairs $\left(q_{k}, h_{k}\right)$, the above proposition shows that the control policy $\theta$ has no effect on the power cost. Thus we can rewrite 
the objective in (8) as

$$
J(\pi, \theta)=\limsup _{N \rightarrow \infty} \frac{1}{N} J_{\mathrm{LQR}}^{N}(\pi, \theta)+\lambda \limsup _{N \rightarrow \infty} \frac{1}{N} J_{\mathrm{PWR}}^{N}(\pi) .
$$

This means that the optimal control policy $\theta \in \Theta$ for a given communication policy $\pi \in \Pi$ is the one minimizing the limit LQR cost. It turns out that the form of the optimal controller does not depend on the communication policy, leading to a stronger separability than what follows from (19).

Indeed by the above separation principle standard dynamic programming arguments show that the optimal control law for a finite horizon is given by the standard LQR one, as in, e.g., [19], [20]. We are interested however in the infinite horizon problem. Our setup differs from the standard LQG/Kalman filtering problem with state observations $y_{k}$ containing Gaussian noise, where the estimation error covariance $\Sigma_{k}$ converges to some limit and the system is assumed to start at time $k=0$ with this limit estimation error. In our setup whenever a packet is received the estimation error is reset to zero otherwise it grows (cf. (17)), so for the general communication policies $\pi \in \Pi$ under consideration it is not clear whether some limit covariance exists. Alternatively the following proposition shows that estimation errors admit a uniform bound in second moment.

Proposition 2. Suppose Assumption 1 holds. Then there exists a finite positive constant $M$ such that for any communication policy $\pi \in \Pi$ selecting successful decoding probabilities $q_{k}$ with respect to $F_{k}$ given in (13), possibly randomized, satisfying the additional restriction $q_{k} \in Q\left(\varepsilon_{k}, h_{k}\right)$ given by (15), and for every $k=0,1, \ldots$, it holds that

$$
\mathbb{E}^{\pi} e_{k}^{T} e_{k} \leq M
$$

Proof: See Appendix A

With this bound on expected magnitude of estimation error established, uniform over $k$ and over any policy $\pi \in \Pi$, the following theorem determines the optimal control law for the average infinite horizon problem (16).

Theorem 1 (Optimal control policy). Consider the wireless control system of Fig. 4 with any communication policy $\pi:=$ $\left\{q_{0}, q_{1}, \ldots\right\} \in \Pi$ selecting successful decoding probabilities $q_{k}$ as functions of $F_{k}$ given in (13), possibly randomized, with innovation terms $\varepsilon_{k}$ as defined in (12) and channel states $h_{k}$ independently drawn from a distribution $m_{H}$, satisfying the additional restriction $q_{k} \in Q\left(\varepsilon_{k}, h_{k}\right)$ given by (15). Suppose Assumption 1 holds. Then for any control policy $\theta:=\left\{u_{0}, u_{1}, \ldots\right\} \in \Theta$ composed of inputs $u_{k}$ as possibly randomized functions of $G_{k}$ in (10) such that

$$
\lim _{N \rightarrow \infty} 1 / N \mathbb{E}^{\pi, \theta} x_{N}^{T} x_{N}=0,
$$

the joint objective $J(\pi, \theta)$ described by (6) - (8) satisfies

$J(\pi, \theta) \geq \operatorname{Tr}(P W)+\limsup _{N \rightarrow \infty} \frac{1}{N} \mathbb{E}^{\pi} \sum_{k=0}^{N-1} e_{k}^{T} \tilde{P} e_{k}+\lambda p\left(h_{k}, q_{k}\right)$

where $P$ is the solution to the standard algebraic Riccati equation $P=A^{T} P A+Q-A^{T} P B\left(R+B^{T} P B\right)^{-1} B^{T} P A$ for the system in (1) and the linear quadratic regulator cost (LQR) in (6), and the matrix $\tilde{P}$ is defined as

$$
\tilde{P}:=A^{T} P A+Q-P .
$$

Moreover, the minimum value in (22) is achieved for the control policy

$$
u_{k}=K \hat{x}_{k}
$$

with $\hat{x}_{k}$ the state estimate described in (11) and the steady state LQR gain $K:=-\left(R+B^{T} P B\right)^{-1} B^{T} P A$.

Proof: See Appendix B

The theorem determines the optimal control policy $\theta$ of problem (16) as the conventional LQR controller in (24), shown in Fig. 4. The optimal cost given in (22) equals a constant $\operatorname{Tr}(P W)$ and a limit average sum term that only depends on the communication policy $\pi \in \Pi$. This term shows that the optimal communication policy needs to balance the power expenditures with a weighted version of the estimation error at the controller.

Observe that as per (11) and (17) it holds that $e_{k}=(1-$ $\left.\gamma_{k}\right) \varepsilon_{k}$. Also $\mathbb{E}^{\pi}\left[\gamma_{k} \mid F_{k}\right]=\mathbb{P}^{\pi}\left[\gamma_{k}=1 \mid F_{k}\right]=q_{k}$ and $\varepsilon_{k} \in F_{k}$. So we can write

$$
\mathbb{E}^{\pi}\left[e_{k}^{T} \tilde{P} e_{k} \mid F_{k}\right]=\mathbb{E}^{\pi}\left[\left(1-\gamma_{k}\right) \varepsilon_{k}^{T} \tilde{P} \varepsilon_{k} \mid F_{k}\right]=\left(1-q_{k}\right) \varepsilon_{k}^{T} \tilde{P} \varepsilon_{k},
$$

and taking the expectation in both sides gives

$$
\mathbb{E}^{\pi}\left[e_{k}^{T} \tilde{P} e_{k}\right]=\mathbb{E}^{\pi}\left[\left(1-q_{k}\right) \varepsilon_{k}^{T} \tilde{P} \varepsilon_{k}\right]
$$

Substituting the expression (26) into the second summand of (22) it follows that the optimal communication policy $\pi \in \Pi$ of problem (16) is the one achieving the infimum cost

$$
J_{\text {COMM }}^{*}:=\inf _{\pi \in \Pi} \lim _{N \rightarrow \infty} \frac{1}{N} \mathbb{E}^{\pi} \sum_{k=0}^{N-1} c\left(\varepsilon_{k}, h_{k}, q_{k}\right),
$$

where we define

$$
c(\varepsilon, h, q):=(1-q) \varepsilon^{T} \tilde{P} \varepsilon+\lambda p(h, q) .
$$

The difference between the sum in (22) and the objective in (27) is that in the former $e_{k}$ is not known at the sensor at time $k$, while $\varepsilon_{k}$ in the latter is. This way (27) takes the form of a Markov decision process (MDP) problem with an infinite horizon average cost criterion. The state of the problem at time $k$ is the pair $\left(\varepsilon_{k}, h_{k}\right) \in \mathbb{R}^{n} \times \mathbb{R}_{+}$, the available action is $q_{k} \in Q\left(\varepsilon_{k}, h_{k}\right)$ by (15), and the cost-per-stage is $c\left(\varepsilon_{k}, h_{k}, q_{k}\right)$. The state transition probabilities can be obtained from (18) and are given by

$$
\begin{aligned}
& \mathbb{P}\left(\varepsilon^{+}, h^{+} \mid \varepsilon, h, q\right) \\
& =\left[q \mathcal{N}_{0, W}\left(\varepsilon^{+}\right)+(1-q) \mathcal{N}_{A \varepsilon, W}\left(\varepsilon^{+}\right)\right] m_{H}\left(h^{+}\right) .
\end{aligned}
$$

Here $\varepsilon, h$ and $\varepsilon^{+}, h^{+}$denote the current and next states respectively, and $q$ the current action. When $q$ is chosen at state $(\varepsilon, h)$, a variable $\gamma \sim \operatorname{Bern}(q)$ is drawn. By (18) on the event $\gamma=1, \varepsilon^{+}=w \sim \mathcal{N}_{0, W}$, while on the event $\gamma=0, \varepsilon^{+}=A \varepsilon+w$ with $w \sim \mathcal{N}_{0, W}$, which is equivalent to $\varepsilon^{+} \sim \mathcal{N}_{A \varepsilon, W}$. Since $h^{+}$is independent of $\varepsilon, h, \varepsilon^{+}$, its distribution $m_{H}$ appears as a product in (29). We denote 
$\mathbb{E}\left[\varepsilon^{+}, h^{+} \mid \varepsilon, h, q\right]$ the integration with respect to the above transition probability measure.

To sum up, we have exploited the proposed decoupling information structure to determine the optimal control policy as the standard LQR control input. We proceed in the following section to show that an optimal communication policy exists and we characterize its main features in the case of general FEC codes and in the special case of capacity achieving codes.

Remark 5. The technical condition (21) for the controller in Theorem 1 can be viewed as an additional stability condition requiring that the norm of the plant state grows at a sub-linear rate. Such conditions appear in general average cost optimal control problems, see e.g. [29, Vol.II, p.254-5], and have also been used in average LQG problems [29, Vol.II, p.272-3]. This technical condition may potentially be relaxed under a different proof technique.

\section{Optimal Communication Policy}

Exploiting the MDP formulation of (27) we can show that optimal communication policies for the co-design problem in (16) exist. This existence result provides a characterization of these policies from which we infer the general features of optimal transmit powers $p_{k}$ and corresponding successful decoding probabilities $q_{k}$ as a function of innovation terms $\varepsilon_{k}$ and channel realizations $h_{k}$.

The existence of optimal policies for average infinitehorizon MDPs on general state spaces requires some technical conditions [30]. In our case restriction to communication policies $\pi \in \Pi$ that uniformly satisfy (15) guarantee existence, as the following theorem shows.

Theorem 2 (Optimal communication policy). Consider the Markov decision process with optimal cost as in (27), state transition probabilities as in (29), and actions restricted to $q_{k} \in Q\left(\varepsilon_{k}, h_{k}\right)$ with $Q(\varepsilon, h)$ abiding to (15). If Assumptions 1 and 2 hold true there exists a function $V: \mathbb{R}^{n} \times \mathbb{R}_{+} \mapsto \mathbb{R}$ such that for all $\varepsilon \in \mathbb{R}^{n}$ and $h \in \mathbb{R}_{+}$it satisfies

$$
\begin{aligned}
V(\varepsilon, h)=\min _{q \in Q(\varepsilon, h)}\left\{c(\varepsilon, h, q)-J_{\text {COMM }}^{*}\right. \\
\left.+\mathbb{E}\left[V\left(\varepsilon^{+}, h^{+}\right) \mid \varepsilon, h, q\right]\right\} .
\end{aligned}
$$

The optimal communication cost can be written as $J_{\text {COMM }}^{*}=$ $\mathbb{E}_{w, h} V(w, h)$, where $\mathbb{E}_{w, h}$ denotes integration with respect to the product measure $\mathcal{N}_{0, W} \times m_{H}$. The optimal communication policy $\pi^{*}$ achieving the minimum cost can be written as a function of the error and channel states at time $k, q_{k}^{*}=q^{*}\left(\varepsilon_{k}, h_{k}\right)$, and is the one achieving the minimum in the right hand side of (30), i.e.

$$
\begin{aligned}
q^{*}(\varepsilon, h):=\underset{q \in Q(\varepsilon, h)}{\operatorname{argmin}}\left\{c(\varepsilon, h, q)-J_{\text {COMM }}^{*}\right. \\
\left.+\mathbb{E}\left[V\left(\varepsilon^{+}, h^{+}\right) \mid \varepsilon, h, q\right]\right\} .
\end{aligned}
$$

Proof: See Appendix C.

The theorem states that the optimal communication policy exists, is deterministic, and also stationary in the sense that $q_{k}^{*}$ adapts only to the current state $\left(\varepsilon_{k}, h_{k}\right)$ and not the complete history $F_{k}$ in (13). The optimal policy is described by (31) in terms of a function $V(\varepsilon, h)$ that solves (30).
Note that this function is unique up to a constant. Related characterizations of optimal communication policies when the decision is whether to transmit or not appear in [15], [16]. Our setup however differs since the decision is on the transmit power and this depends on the random wireless channel state. The proof of the theorem relies on constructing a Lyapunovlike function that is common for all policies $\pi \in \Pi$, and applying the MDP results of [31]. This methodology has been used in [16], however a refined construction is required here to account for the random channel states.

An informal interpretation of condition (30) based on finite state spaces [29] is the following. Suppose the constant $J_{\text {COMM }}^{*}$, corresponding to the optimal cost of (27), was known. Subtracting this constant from the cost-per-stage in problem (27) does not change the optimal policy, and gives a relative cost per stage $c(\varepsilon, h, q)-J_{\text {COMM }}^{*}$ indicating how far we are from the optimal average cost. Then equation (30) has exactly the form of a standard Bellman equation for a nonaveraged infinite horizon problem with this relative cost per stage [29, Vol.I, Ch.7]. The function $V(\varepsilon, h)$ captures the expected future relative cost of following the optimal policy when starting from state $(\varepsilon, h)$, and is termed the relative value function. Bellman's equation (30) states that the optimal choice $q$ at every step minimizes the sum of the current-stage relative cost $c(\varepsilon, h, q)-J_{\mathrm{COMM}}^{*}$ and the expected future relative cost $\mathbb{E}\left[V\left(\varepsilon^{+}, h^{+}\right) \mid \varepsilon, h, q\right]$. The minimization over the current action $q$ gives again the value $V(\varepsilon, h)$ of the current state at the left hand side of (30).

In principle one can find $V(\varepsilon, h)$ using value iteration or policy iteration algorithms which involve iterative application of (30) [30]. This procedure is, however, computationally onerous as each iteration requires minimizing the right hand side of (30) for all possible state pairs $(\varepsilon, h) \in \mathbb{R}^{n} \times \mathbb{R}_{+}$. Nevertheless, (30) still gives qualitative information on the optimal policy.

Let us ignore the case $\|\varepsilon\| \geq L, h \geq h_{t}$ in (15) as it is irrelevant for the following discussion. Integrating $V(\varepsilon, h)$ with respect to the transition (29) gives

$$
\begin{aligned}
& \mathbb{E}\left[V\left(\varepsilon^{+}, h^{+}\right) \mid \varepsilon, h, q\right] \\
& =q \mathbb{E}_{w, h^{+}} V\left(w, h^{+}\right)+(1-q) \mathbb{E}_{w, h^{+}} V\left(A \varepsilon+w, h^{+}\right) .
\end{aligned}
$$

We substitute this, the cost-per-stage $c(\varepsilon, h, q)$ defined by (28), and the expression $J_{\mathrm{COMM}}^{*}=\mathbb{E}_{w, h} V(w, h)$ provided by the theorem in the minimization of (31), and upon reordering terms, the optimal communication policy can be written as

$$
q^{*}(\varepsilon, h)=\underset{q \in\left[0, q_{\max }(h)\right]}{\operatorname{argmin}} \lambda p(h, q)+(1-q) R(\varepsilon),
$$

where for convenience we defined the function

$$
R(\varepsilon):=\mathbb{E}_{w, h}[V(A \varepsilon+w, h)-V(w, h)]+\varepsilon^{T} \tilde{P} \varepsilon,
$$

which can be thought as a penalty function on the error $\varepsilon$. The optimal policy $q^{*}(\varepsilon, h)$ depends on the shape of the function $p(h, q)$ and takes values anywhere in the interval $\left[0, q_{\max }(h)\right]$. The optimal power allocation can be found by converting (33) to power by (2), (4), and is described by

$$
p^{*}(\varepsilon, h):=\underset{p \in\left[0, p_{\max }\right]}{\operatorname{argmin}} \lambda p+(1-q(h, p)) R(\varepsilon) .
$$



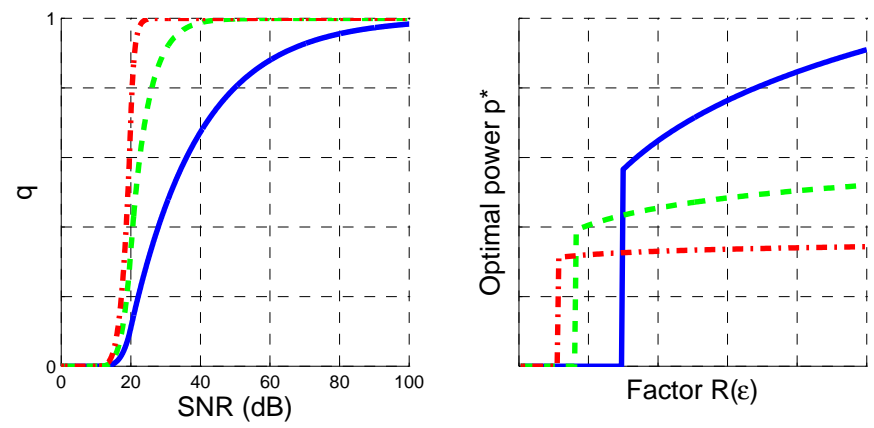

Fig. 5. Optimal power allocation for FEC codes with different complementary error functions. The optimal transmit power $p^{*}$ is plotted as a function of the factor $R(\varepsilon)$ for a fixed channel state $h$ using FEC codes with different $q$-SNR characteristics. When the $q$-SNR curve becomes steeper, the optimal power allocation resembles a step function.

Despite the fact that $V(\varepsilon, h)$ and $R(\varepsilon)$ are hard to compute, the above expression is an important characterization of the optimal power allocation. It provides a tool for qualitative analysis of different FEC codes in wireless control systems. We illustrate this in Fig. 5 where we examine how the $q$ SNR relationship of a FEC code affects the optimal power allocation. For simplicity we fix the channel state $h$ and plot $p^{*}$ in Fig. 5 as a function of $R(\varepsilon)$. In all cases, when the error penalty $R(\varepsilon)$ is below some threshold, the best option is to not transmit. Above the threshold, the optimal transmit power increases as the error penalty $R(\varepsilon)$ gets larger. For powerful FEC codes characterized by a steep $q$-SNR relationship, close to the theoretical limit in (3), the optimal power allocation resembles a step function, since the probability of successful decoding becomes practically one for large powers. For fat $q$-SNR tails, this behavior deteriorates as the sensor needs to transmit with higher power to achieve a larger $q$.

Then in Fig. 6 we present qualitative plots of the optimal decoding probability $q^{*}$ and optimal transmit power $p^{*}$ as functions of both the factor $R(\varepsilon)$ and the channel state $h$ for a given $q$-SNR characteristic. The blue region indicates the event where no transmission occurs. This happens if channel gain $h$ is low, where transmission is costly, or if error $\varepsilon$ has a low penalty, meaning that there is no need to update the receiver's estimate. This no-transmission region becomes larger for a higher penalty $\lambda$ on power in (8). Outside this region a transmission occurs and transmit power adapts to both channel and error states. In principle when channel gain $h$ is high, a small amount of power suffices. For intermediate values of channel $h$ power takes a wide range of values depending on the error as well.

Overall this optimal power management displays different features than the standard "0-1" event-triggered transmission paradigm of, e.g., [15] or [12]. It can be though as a 'soft" version of these policies since the power decision ranges between $\left[0, p_{\max }\right]$, or equivalently the decoding $q$ between $\left[0, q_{\max }(h)\right]$. Finally we note that the transmit power/remote estimation problem has also been studied in the very recent works [23], [24], which however consider only power adaptation to the channel and the packet drop processes, not the plant state/error observed online. Hence the qualitative
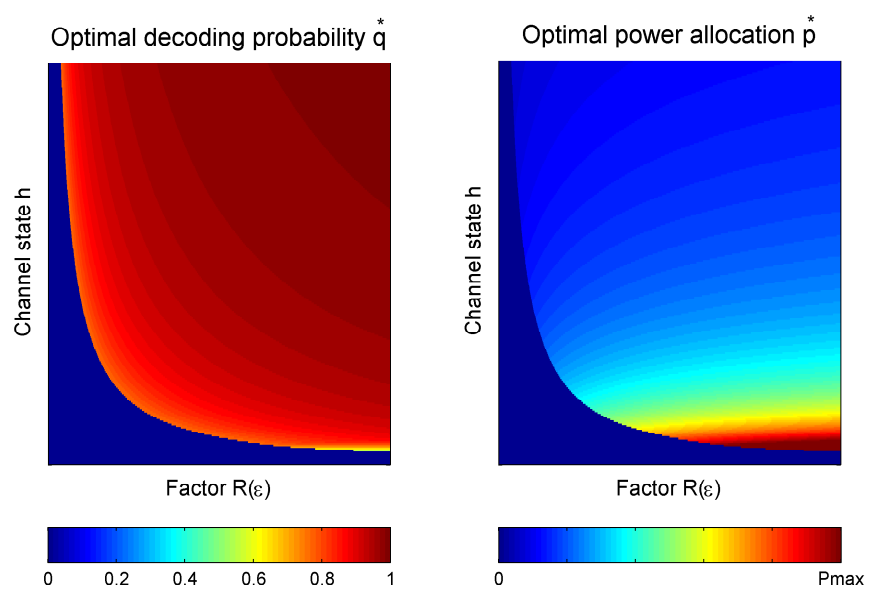

Fig. 6. Optimal decoding probability and power allocation for a FEC code. Color intensity indicates the magnitude of optimal decoding probability $q^{*}$ and optimal transmit power $p^{*}$ as functions of the factor $R(\varepsilon)$ and the channel state $h$.

characterization we discuss here and the connections with the event-triggered paradigm were not apparent.

\section{A. Optimal solution for capacity achieving codes}

Consider now the case of capacity achieving codes. By (3), at time $k$ the transmitter needs to use either $p_{k}=0$, i.e. not transmitting, or $p_{k}=p_{0} / h_{k}$ with $p_{0}:=N_{0} \mathrm{SNR}_{0}$, which certainly guarantees correct packet delivery. Any other power allocation is unfavorable. However the instantaneous power is bounded by $p_{k} \leq p_{\max }$, so the sensor can transmit only when $p_{0} / h_{k} \leq p_{\max }$, or equivalently when the channel state exceeds $h_{k} \geq p_{0} / p_{\max }$.

In this case we are looking again for a randomized policy, i.e. a distribution on the two power options $\left\{0, p_{0} / h_{k}\right\}$ when $h_{k} \geq p_{0} / p_{\max }$. With a slight abuse of notation we denote $q_{k} \in[0,1]$ the probability of choosing power $p_{0} / h_{k}$. Then when $h_{k} \geq p_{0} / p_{\max }$ the transmitter draws independent $\gamma_{k} \sim \operatorname{Bern}\left(q_{k}\right)$ and transmits with power $p_{k}=\gamma_{k} p_{0} / h_{k}$. The decoding success at the receiver is given by the same $\gamma_{k}$. The expected power consumption becomes

$$
\mathbb{E} \sum_{k=0}^{N-1} p_{k}=\mathbb{E} \sum_{k=0}^{N-1} q_{k} \frac{p_{0}}{h_{k}} \mathbb{I}\left(h_{k} \geq \frac{p_{0}}{p_{\max }}\right) .
$$

Observe that this is of the same form as the expected power consumption of the original problem given in (7) with the function $p(h, q)$ substituted with $q p_{0} / h \mathbb{I}\left(h \geq p_{0} / p_{\max }\right)$. Then the statements of the results so far hold for the capacity achieving codes as well. For this special case of $p(h, q)$ however the minimization in (33) becomes linear in $q$, and the optimal communication policy is deterministic,

$$
q^{C A}(\varepsilon, h):= \begin{cases}0 & \text { if } h R(\varepsilon) \leq \lambda p_{0} \text { or } h \leq p_{0} / p_{\max } \\ 1 & \text { otherwise }\end{cases}
$$

or in terms of power

$p^{C A}(\varepsilon, h):=\left\{\begin{array}{ll}0 & \text { if } h R(\varepsilon) \leq \lambda p_{0} \text { or } h \leq p_{0} / p_{\max } \\ p_{0} / h & \text { otherwise }\end{array}\right.$. 
This is an event-triggered transmission scheme along the lines of, e.g., [15], except that now the decision is also affected by the current channel state $h$ apart from the error $\varepsilon$. This deterministic policy was expected as the limit behavior of powerful FEC codes in Fig. 5. The region of the plant/channel state space $\mathbb{R}^{n} \times \mathbb{R}_{+}$where it is optimal to transmit is described in (37) as $h R(\varepsilon)>\lambda p_{0}$ and $h \geq p_{0} / p_{\max }$. Intuitively condition $h R(\varepsilon)>\lambda p_{0}$ states that when channel gain is large, transmitting is worthy as it does not cost much, while when an error penalty $R(\varepsilon)$ is large, it is necessary to transmit in order to reset it to zero. This region gets smaller when the constant $p_{0}=N_{0} \mathrm{SNR}_{0}$ (cf. (3)) increases, since transmission then requires more power, or when $\lambda$ increases, since power then is penalized more in the objective (8).

In the following section we present easily computable suboptimal communication policies, which we examine with simulations in Section VI.

\section{A ROLLOUT COMMUNICATION POLICY}

The optimal communication policy $q^{*}(\varepsilon, h)$ was described in the previous section in terms of the relative value function $V(\varepsilon, h)$ which is not computationally tractable in general. The purpose of this section is to show how approximate dynamic programming can be used to devise tractable suboptimal policies.

As shown in Theorem 2, the optimal communication policy $q^{*}(\varepsilon, h)$ given by (31) selects the decision $q$ that minimizes a combination of the current cost $c(\varepsilon, h, q)$ and the optimal expected future cost $\mathbb{E}\left[V\left(\varepsilon^{+}, h^{+}\right) \mid \varepsilon, h, q\right]$. However the function $V(\varepsilon, h)$ is not available. Suppose instead that some suboptimal communication policy $\pi$ is available, for which the corresponding relative value function $V^{\pi}(\varepsilon, h)$ is known at all state pairs $(\varepsilon, h)$. This means that if the transmitter employs this given policy $\pi$ at all future steps, we can model the expected future cost induced by $\pi$ as $\mathbb{E}\left[V^{\pi}\left(\varepsilon^{+}, h^{+}\right) \mid \varepsilon, h, q\right]$. Then the optimal current action under this suboptimal model of future communication decisions is described by

$q^{\text {roll }}(\varepsilon, h):=\underset{q \in\left[0, q_{\max }(h)\right]}{\operatorname{argmin}} c(\varepsilon, h, q)+\mathbb{E}\left[V^{\pi}\left(\varepsilon^{+}, h^{+}\right) \mid \varepsilon, h, q\right]$.

This approximation defines a rollout algorithm [29, Vol. I].

We consider a family of suboptimal policies of the form $q_{k}=q\left(h_{k}\right)$ adapting only to channel state $h_{k}$ and not to innovation $\varepsilon_{k}$, which have a computable relative value function. Policies of this form have been proposed in prior works [23], [24]. Since channel states are independent of $\varepsilon_{k}$ the policy $q(h)$ results in successful packet decodings with expected probability $\bar{q}:=\mathbb{E}_{h} q(h)$ implying that the communication success indicator variable is $\gamma_{k} \sim \operatorname{Bern}(\bar{q})$. The expected power consumption at every stage is constant given by $\mathbb{E}_{h} p(h, q(h))$. Thus the cost of this policy $q(h)$ for the MDP problem in (27) becomes

$$
J^{q(\cdot)}:=\limsup _{N \rightarrow \infty} \frac{1}{N} \mathbb{E} \sum_{k=0}^{N-1}(1-\bar{q}) \varepsilon_{k}^{T} \tilde{P} \varepsilon_{k}+\lambda \mathbb{E}_{h} p(h, q(h)) .
$$

For any policy of the form $q_{k}=q\left(h_{k}\right)$ the corresponding relative value function $V^{q(\cdot)}(\varepsilon, h)$ can be determined in closed form as stated in the following theorem that also provides an explicit expression for the cost $J^{q(\cdot)}$.

Theorem 3 (Cost of channel-adaptive communication policies). Consider the Markov decision process with state pair $(\varepsilon, h)$ and state transition probabilities as in (29). Consider policies $q(\cdot)$ for which the success transmission probability is selected as a function $q(h)$ independent of the innovation terms $\varepsilon$. For any policy of this form satisfying $\bar{q}:=\mathbb{E}_{h} q(h)>q_{\text {crit }}$ for the critical probability $q_{\text {crit }}$ of Assumption 1, the cost $J^{q(\cdot)}$ in (40) becomes

$$
J^{q(\cdot)}=\operatorname{Tr}(\tilde{P} E)+\lambda \mathbb{E}_{h} p(h, q(h)),
$$

where the matrix $E$ is the unique solution of

$$
E=(1-\bar{q})\left(A E A^{T}+W\right) .
$$

Furthermore, the relative value function $V^{q(\cdot)}$ is given by

$$
V^{q(\cdot)}(\varepsilon, h)=\frac{1-q(h)}{1-\bar{q}} \varepsilon^{T} H \varepsilon+\lambda p(h, q(h)),
$$

where the matrix $H$ is the unique solution of

$$
H=(1-\bar{q})\left(A^{T} H A+\tilde{P}\right) .
$$

Proof: See Appendix D.

Theorem 3 provides an explicit formula for a family of relative value functions $V^{q(\cdot)}(\varepsilon, h)$ that can be used in the rollout algorithm in (39). Substituting (43) into (39) and removing constants from the resulting expression we find the rollout policy

$$
q^{\mathrm{roll}}(\varepsilon, h):=\underset{q \in\left[0, q_{\max }(h)\right]}{\operatorname{argmin}} \lambda p(h, q)+(1-q) \frac{\varepsilon^{T} H \varepsilon}{1-\bar{q}} .
$$

Computing such policies is easy. Given the parameter $\bar{q}$ that models the suboptimal future actions, we can compute $H$ by (44) and then solve (45) given the function $p(h, q)$. Observe that (45) is of the same form as the optimal communication policy (33) except that the optimal unknown function $R(\varepsilon)$ is replaced by the quadratic form $\varepsilon^{T} H \varepsilon /(1-\bar{q})$. Since the rollout policy is suboptimal the quadratic can be viewed as an approximation of the function $R(\varepsilon)$. As a side note, the rollout policy need not satisfy the technical requirement $q^{\text {roll }}(\varepsilon, h) \in$ $Q(\varepsilon, h)$ of (15).

For the particular case of capacity achieving codes repeating the analysis of Section IV-A to modify (45) we obtain the suboptimal policy

$$
q^{\text {roll }, C A}(\varepsilon, h):= \begin{cases}0 & \text { if } h \frac{\varepsilon^{T} H \varepsilon}{1-\bar{q}} \leq \lambda p_{0} \text { or } h \leq \frac{p_{0}}{p_{\max }} \\ 1 & \text { otherwise }\end{cases}
$$

Again the unknown function $R(\varepsilon)$ in (37) is approximated by a quadratic that can be computed by (44). This is an explicit event triggered communication policy, where the events depend on the current channel state $h$ and error $\varepsilon$.

The rollout policy (39) is a heuristic. Intuitively if the reference policy $\pi$ is close to the optimal policy then the rollout is close to the optimal as well. It is not easy to 

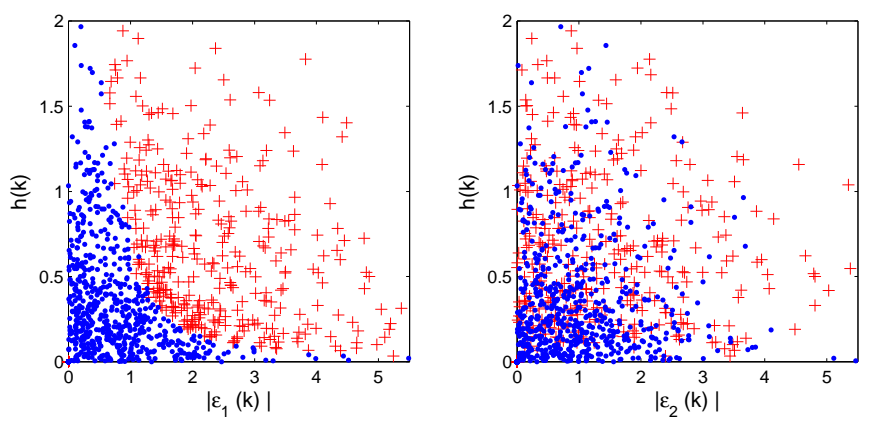

Fig. 7. Simulation results of the rollout policy for a capacity achieving code. The points $\left|\varepsilon_{k, 1}\right|, h_{k}$ and $\left|\varepsilon_{k, 2}\right|, h_{k}$ are plotted respectively, with blue dots indicating the decision to not transmit, $q_{k}=0$, and red crosses the decision to transmit, $q_{k}=1$. The decision is highly dependent on $\varepsilon_{k, 1}$ corresponding to the unstable dynamics of the plant.

characterize how worse the rollout performs compared to the optimal value $J_{\text {COMM }}^{*}$. However it is guaranteed by design to perform not worse than the reference policy $\pi$. In many practical problems the improvement is significant [29, Vol. I]. In the following section we simulate the constructed rollout policies and characterize numerically the improvement to the reference policies adapting only to channel.

\section{Simulations}

We begin by presenting simulations of the rollout algorithm for capacity achieving codes given in (46). We assume the channel state distribution to be exponential with mean 0.5. The plant is given by

$$
A=\left[\begin{array}{cc}
2 & 0 \\
1 & 0.8
\end{array}\right], B=\left[\begin{array}{l}
2 \\
1
\end{array}\right] .
$$

The rest of the parameters are $Q=W=I, R=1$, $\lambda=50, p_{0}=1$. As a reference communication policy we use $q(h)=\mathbb{I}\left(h \geq h_{t}\right)$ which transmits whenever the channel state is above some threshold $h_{t}$, inducing a constant transmission rate $\bar{q} \approx 0.79$. The simulations of the rollout policy reveal a dramatic decrease in the empirical transmission rate $q_{e m p}=1 / N \sum_{k=0}^{N-1} \gamma_{k} \approx 0.37$, which is also much lower than the minimum non-adaptive transmission rate $q_{\text {crit }}=0.75$ that would keep the error stable (cf. (5), (17)). Similarly, the empirical cost of the rollout policy $J_{\text {emp }}=1 / N \sum_{k=0}^{N-1} e_{k}^{T} \tilde{P} e_{k}+\lambda \gamma_{k} p_{0} / h_{k} \approx 56$ decreased compared to the reference $J^{q(\cdot)} \approx 124$.

The event-triggered nature of the rollout policy in the case of capacity achieving codes is captured in Fig. 7 where we plot the two plant states $\left|\varepsilon_{k, 1}\right|,\left|\varepsilon_{k, 2}\right|$ along with the channel $h_{k}$ during the simulation. Blue dots indicate the decision not to transmit, $q_{k}=0$, while red crosses are the points where $q_{k}=1$. When the channel fading coefficient $h_{k}$ is low, the sensor avoids transmission as it requires large power consumption. The rollout policy is also adapted to the plant structure. The error state $\varepsilon_{k, 1}$ is related to the unstable eigenvalue of $A$, so the sensor always decides to transmit when this state is far from 0 . The hyperbolic shape of the $\left|\varepsilon_{k, 1}\right|, h_{k}$ plot is justified by the form of the rollout algorithm in (46). In contrast, such a correlation between the error state $\varepsilon_{k, 2}$ and the
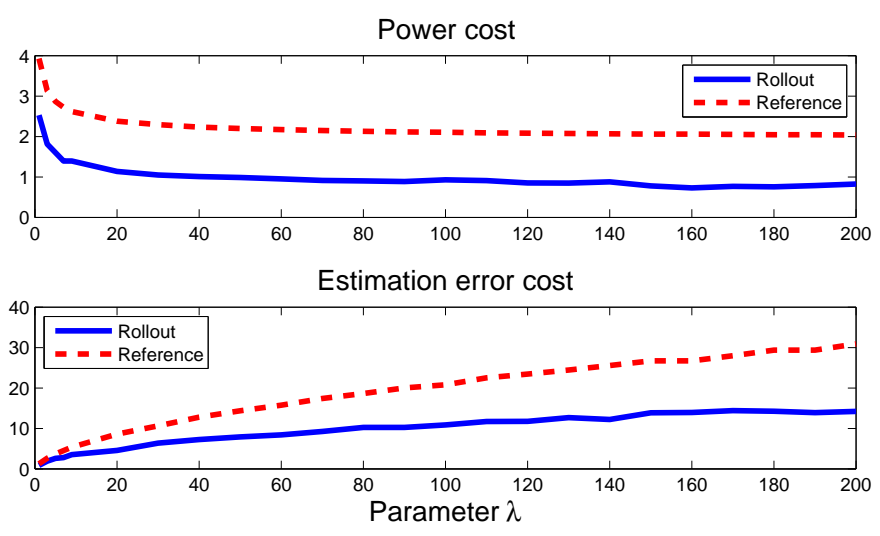

Fig. 8. Comparison of the optimal channel-threshold policy and the resulting rollout policy performance for a capacity achieving code. The power and estimation error costs of the two policies are plotted for different values of the power weight $\lambda$. As the power weight increases, the estimation performance of the reference policy becomes increasingly worse compared to the rollout.

decision to transmit is not clear. Even when $\varepsilon_{k, 2}$ takes large values, the sensor might choose to not transmit. The reason is that this state's dynamics are related to a stable eigenvalue, so informally $\varepsilon_{k, 2}$ will remain bounded even if the sensor takes no action. More precisely, as long as the sensor keeps $\varepsilon_{k, 1}$ bounded, $\varepsilon_{k, 2}$ will also be bounded.

Next, for the plant and channel described above we compare the performance of the rollout algorithm with that of the reference policy we used to compute the value function in (43). For different values of $\lambda$ we find the optimal threshold policy $q(h)=\mathbb{I}\left(h \geq h_{t}\right)$, i.e. the one that minimizes (41), and the corresponding rollout policy in (46). The resulting power cost $1 / N \sum_{k=0}^{N-1} \gamma_{k} p_{0} / h$ and estimation cost $1 / N \sum_{k=0}^{N-1} e_{k}^{T} \tilde{P} e_{k}$ that we got from simulating the rollout algorithm are plotted separately in Fig. 8 along with the costs of the reference policy. As $\lambda$ increases the power consumption decreases, since it is penalized more in the aggregate cost (27), and the decrease rate is similar for the rollout and the reference policies. On the other hand, when $\lambda$ increases the estimation cost increases, since the sensor decides to transmit less often. However the increase for the rollout policy is slower than that of the reference policy. The reason is that the reference only adapts to the channel, avoiding transmissions when the channel state $h_{k}$ is low. The rollout algorithm adapts not only to the channel, but also to the error $\varepsilon_{k}$. By transmitting only when $\varepsilon_{k}$ is large, it results in only a moderate increase in the estimation cost without sacrificing too much power.

Finally, we simulate the rollout algorithm (45) for the above plant and channel model when a FEC code is employed. The probabilities $q_{k}$ of successful decoding that the rollout selected during the simulation are plotted in Fig. 9 on $\left\|\varepsilon_{k}\right\|, h_{k}$ axes (compare with the optimal policy in Fig. 6). Unlike the capacity achieving codes, $q_{k}$ take values smaller than 1 . However, due to the sigmoid form of the $q$-SNR characteristic of the FEC code (cf. Fig. 2), practically $q_{k}$ are either 0 or very close to 1 , especially when the channel state is good $\left(h_{k}\right.$ large). For low channel gain $h_{k}$ a very high power penalty is incurred to transmit with high success probability, hence the 


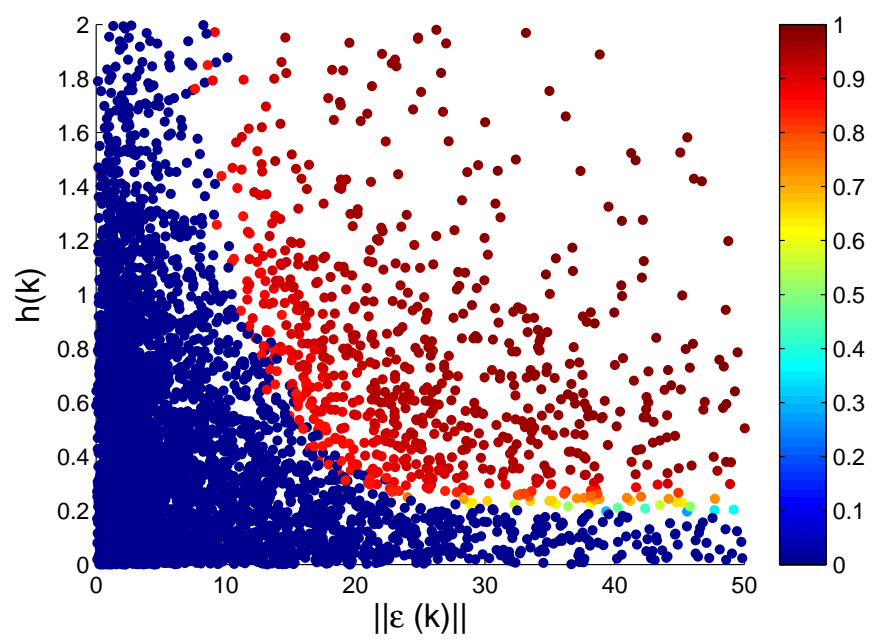

Fig. 9. Simulation results of the rollout policy for a FEC code. The points $\left\|\varepsilon_{k}\right\|, h_{k}$ during the simulation are plotted with colors denoting the magnitude of the chosen decoding probability $q_{k}$. Practically $q_{k}$ takes values either 0 or close to 1 .

rollout chooses either to not transmit $\left(q_{k}=0\right)$ or transmit with $q_{k}$ very close to 1 . The simulation points are accumulated in the region of small values of $\left\|\varepsilon_{k}\right\|$ since when the error gets large, $q_{k}$ is chosen close to 1 and the error gets reset with high probability.

\section{CONCLUSIONS AND FUTURE WORK}

In this paper we examined a control system with a wireless fading channel between the sensor and the controller. The sensor adapts transmit power to plant and channel states and affects the probability of successful decoding at the controller. For the problem of co-designing transmit powers and control inputs to minimize an average LQR and power cost a method to separate the two designs is provided, leading to the standard LQR controller. The optimal power allocation is then characterized qualitatively for general FEC codes and capacity achieving codes. Tractable suboptimal policies are derived and their performance is compared with alternative policies in simulations.

Near-optimal and computationally efficient policies for the general co-design framework requires further research. For example power policies adapting to the plant state directly, not the estimation innovation, need to be examined. Further work includes also the incorporation of other wireless channel models, e.g., Markov. Moreover interference effects when multiple control loops close over the same channel need to be considered. Overall the proposed framework can be expanded to accommodate modeling and analysis of more complex wireless sensor \& actuator networks. This unified control/wireless networking framework could serve in novel design problems, such as a control-aware network resource allocation, or a resource-aware networked controller synthesis.

\section{APPENDIX}

\section{A. Proof of Proposition 2}

First note that, by the same arguments we use to derive (26) later, conditioned on $F_{k}$ we can rewrite

$$
\mathbb{E}^{\pi} e_{k}^{T} e_{k}=\mathbb{E}^{\pi}\left(1-q_{k}\right) \varepsilon_{k}^{T} \varepsilon_{k}
$$

The bound in (20) will be shown by an equivalent bound on the innovation process $\left\{\varepsilon_{k}, k \geq 0\right\}$. By Proposition 1 for any communication policy $\pi \in \Pi$ this process is independent of the control policy $\theta \in \Theta$ and its evolution is given by (18). This evolution can be described more formally along with the i.i.d. channel process $h_{k} \sim m_{H}$ by a stochastic transition kernel given the values of $\varepsilon, h$ and decision $q$ at each step as

$$
\begin{aligned}
& \mathbb{P}\left(\varepsilon^{+}, h^{+} \mid \varepsilon, h, q\right) \\
& =\left[q \mathcal{N}_{0, W}\left(\varepsilon^{+}\right)+(1-q) \mathcal{N}_{A \varepsilon, W}\left(\varepsilon^{+}\right)\right] m_{H}\left(h^{+}\right) .
\end{aligned}
$$

This expression is included again in (29), where its derivation is explained in detail.

The following technical lemma shows that under Assumption 1 one can construct a Lyapunov-like function common for all communication policies, satisfying explicitly the technical requirements of [31, Assumptions 3.1, 3.2]. The uniform bound (20) will be a direct consequence of these requirements. We note also that the lemma will be subsequently used to prove Theorem 2 based on the results of [31].

Lemma 1. Suppose Assumption 1 holds and consider the innovation and channel processes $\left\{\varepsilon_{k}, h_{k}, k \geq 0\right\}$ described by the transition (49), with communication decisions satisfying $q_{k} \in Q\left(\varepsilon_{k}, h_{k}\right)$ given in (15). Then there exists a measurable function $W$ on $\mathbb{R}^{n} \times \mathbb{R}_{+}$bounded below by a constant $\gamma>0$ such that

$$
(1-q) \varepsilon^{T} \varepsilon+c \leq K W(\varepsilon, h),
$$

where $c \geq 0$ is some constant, for all $\varepsilon, h \in \mathbb{R}^{n} \times \mathbb{R}_{+}, q \in$ $Q(\varepsilon, h)$, for some positive $K$. Moreover there exists a nontrivial measure $\nu$ on $\mathbb{R}^{n} \times \mathbb{R}_{+}$, a non-negative measurable function $\phi(\varepsilon, h, q)$ for $\varepsilon, h \in \mathbb{R}^{n} \times \mathbb{R}_{+}, q \in Q(\varepsilon, h)$, and $a$ positive constant $\mu<1$ such that

$$
\begin{aligned}
& \text { (i) } \nu(W):=\int W(\varepsilon, h) d \nu(\varepsilon, h)<\infty, \\
& \text { (ii) } \mathbb{P}\left(\varepsilon^{+} \in B_{1}, h^{+} \in B_{2} \mid \varepsilon, h, q\right) \geq \nu\left(B_{1}, B_{2}\right) \phi(\varepsilon, h, q)
\end{aligned}
$$

for all measurable subsets $\left(B_{1}, B_{2}\right) \in \mathcal{B}\left(\mathbb{R}^{n} \times \mathbb{R}_{+}\right)$,

(iii) $\mathbb{E}\left[W\left(\varepsilon^{+}, h^{+}\right) \mid \varepsilon, h, q\right] \leq \mu W(\varepsilon, h)+\phi(\varepsilon, h, q) \nu(W)$

(iv) $\int \phi(\varepsilon, h, q) d \nu(\varepsilon, q)>0$ for all $q \in Q(\varepsilon, h)$.

Proof: The proof is constructive. Let

$$
\nu:=\mathcal{N}_{0, W} \times m_{H} \text { and } \phi(\varepsilon, h, q):=q .
$$

Let us denote the set where the choice of $q$ is free as

$$
S:=\left\{(\varepsilon, h) \in \mathbb{R}^{n} \times \mathbb{R}_{+}:\|\varepsilon\|<L \text { or } h<h_{t}\right\} .
$$

We choose $\mu<1$ such that

$$
\begin{aligned}
& \mu>1-q_{\max }\left(h_{t}\right)+q_{\max }\left(h_{t}\right) \nu(S), \\
& \mu>(1-\bar{q}) \lambda_{\max }(A)^{2}
\end{aligned}
$$


where $\bar{q}$ denotes the integral introduced in (14),

$$
\bar{q}:=\int_{h_{t}}^{+\infty} q_{\max }(h) d m_{H}(h) .
$$

The right hand side of (53) is less than 1 because the event $S$ under the measure $\nu$ happens with probability less than 1 and we have assumed $q_{\max }\left(h_{t}\right)>0$. The right hand side of (54) is also less than 1 because of Assumption 1 and by the choice for $h_{t}$ that satisfies (14).

For future reference note that by construction of the set $Q(\varepsilon, h)$, for any $L>0$, when $\|\varepsilon\| \geq L$ we can upper bound

$$
1-q \leq 1-q_{\max }(h) \mathbb{I}\left(h \geq h_{t}\right)=: \psi(h),
$$

where we named the quantity on the right $\psi(h)$ to be used within this proof. This inequality holds because when $h<h_{t}$, we have $q \geq 0$, and when $h \geq h_{t}$, we choose $q=q_{\max }(h)$.

Finally we pick

$$
W(\varepsilon, h):=\psi(h) \varepsilon^{T} H \varepsilon+\beta \mathbb{I}(\varepsilon, h \in S)+\gamma,
$$

where $\beta, \gamma>0$ are appropriate positive constants that will be designed next, and $H>0$ is a positive definite matrix satisfying

$$
(1-\bar{q}) A^{T} H A-\mu H=-\Theta,
$$

for some positive definite matrix $\Theta>0$. This Lyapunov equation is feasible by our choice of $\mu$ that satisfies (54).

Next we show that the conditions of the lemma are satisfied for the constructed quantities. First observe that $W(\varepsilon, h) \geq$ $\gamma>0$ by construction. Then we check (50). When $\|\varepsilon\|<L$,

$$
(1-q) \varepsilon^{T} \varepsilon+c \leq L^{2}+c \leq K(\beta+\gamma) \leq K W(\varepsilon, h),
$$

for a sufficiently large $K$, where the last inequality follows from the form of $W(\varepsilon, h)$ on $\|\varepsilon\|<L$. On the other hand if $\|\varepsilon\| \geq L$, we may use (56) to upper bound

$$
\begin{aligned}
(1-q) \varepsilon^{T} \varepsilon+c & \leq \psi(h) \varepsilon^{T} \varepsilon+c \\
& \leq K\left(\psi(h) \varepsilon^{T} H \varepsilon+\gamma\right) \leq K W(\varepsilon, h),
\end{aligned}
$$

for a sufficiently large $K$, by our choice for the function $W(\varepsilon, h)$ when $\|\varepsilon\| \geq L$.

We proceed to show that parts (i)-(iv) in the statement of the lemma also hold. Part (i) holds because the integral of $W(\varepsilon, h)$ with our chosen measure $\nu$ equals

$$
\nu(W)=(1-\bar{q}) \operatorname{Tr}(H W)+\nu(S) \beta+\gamma<\infty .
$$

Part (ii) holds because the transition probability in (49) gives

$$
\begin{aligned}
& \mathbb{P}\left(\varepsilon^{+} \in B_{1}, h^{+} \in B_{2} \mid \varepsilon, h, q\right) \\
& =\left[q \mathcal{N}_{0, W}\left(B_{1}\right)+(1-q) \mathcal{N}_{A \varepsilon, W}\left(B_{1}\right)\right] m_{H}\left(B_{2}\right) \\
& \geq q \mathcal{N}_{0, W}\left(B_{1}\right) m_{H}\left(B_{2}\right)=\phi(\varepsilon, h, q) \nu\left(B_{1}, B_{2}\right) .
\end{aligned}
$$

Part (iv) follows by our choice $\phi(\varepsilon, h, q)=q$ and the construction of the set $Q(\varepsilon, h)$ in (15) because

$$
\begin{aligned}
& \int \phi(\varepsilon, h, q) d \nu(\varepsilon, h) \geq \int_{\varepsilon, h \in S^{c}} q_{\max }(h) d \nu(\varepsilon, h) \\
& =\bar{q} \int_{\|\varepsilon\| \geq L} d \mathcal{N}_{0, W}(\varepsilon)>0 .
\end{aligned}
$$

The remainder of the proof shows that (iii) also holds. First observe that by the transition defined in (49) and our choices for $\nu$ and $\phi$ we have

$$
\begin{aligned}
& \mathbb{E}\left[W\left(\varepsilon^{+}, h^{+}\right) \mid \varepsilon, h, q\right]=\phi(\varepsilon, h, q) \nu(W) \\
& +(1-q) \int W\left(\varepsilon^{+}, h^{+}\right) d \mathcal{N}_{A \varepsilon, W}\left(\varepsilon^{+}\right) d m_{H}\left(h^{+}\right) .
\end{aligned}
$$

Substituting (64) in (iii), we only need to show that

$$
(1-q) \int W\left(\varepsilon^{+}, h^{+}\right) d \mathcal{N}_{A \varepsilon, W}\left(\varepsilon^{+}\right) d m_{H}\left(h^{+}\right) \leq \mu W(\varepsilon, h) .
$$

Plugging the expression of $W(\varepsilon, h)$ given by (57) in the integral of the left hand side, condition (65) becomes

$$
\begin{aligned}
(1-q)\{ & (1-\bar{q})\left[\varepsilon^{T} A^{T} H A \varepsilon+\operatorname{Tr}(H W)\right] \\
& \left.+\beta \mathcal{N}_{A \varepsilon, W} \times m_{H}(S)+\gamma\right\} \leq \mu W(\varepsilon, h) .
\end{aligned}
$$

We can bound $\mathcal{N}_{A \varepsilon, W} \times m_{H}(S) \leq \nu(S)$ for any $\varepsilon \in \mathbb{R}^{n}$, and also $(1-q)(1-\bar{q}) \operatorname{Tr}(H W) \leq \operatorname{Tr}(H W)$. So a sufficient condition for (66) is to show that

$$
\begin{aligned}
& (1-q)\left\{(1-\bar{q}) \varepsilon^{T} A^{T} H A \varepsilon+\beta \nu(S)+\gamma\right\}+\operatorname{Tr}(H W) \\
& \leq \mu W(\varepsilon, h)
\end{aligned}
$$

holds for every choice of $q \in Q(\varepsilon, h)$. To show this we examine cases.

Case $\|\varepsilon\| \geq L$. Using (56) to upper bound $1-q \leq \psi(h)$, and upon substituting $W(\varepsilon, h)$ in (67) and rearranging terms, we need to show equivalently that

$$
\begin{aligned}
& \psi(h)\left\{\varepsilon^{T}\left[(1-\bar{q}) A^{T} H A-\mu H\right] \varepsilon\right. \\
& +\beta \nu(S)+\gamma\}+\operatorname{Tr}(H W) \leq \mu\left\{\beta \mathbb{I}\left(h<h_{t}\right)+\gamma\right\}
\end{aligned}
$$

By the choice of $H$ in (58) the quadratic on the left hand side is negative definite equal to to $-\varepsilon^{T} \Theta \varepsilon$. And since $\|\varepsilon\| \geq L$ we can upper bound $-\varepsilon^{T} \Theta \varepsilon \leq-\lambda_{\min }(\Theta) L^{2} \leq 0$. After these, a sufficient condition for (68) is

$$
\operatorname{Tr}(H W)+\psi(h)\{\beta \nu(S)+\gamma\} \leq \mu\left\{\beta \mathbb{I}\left(h<h_{t}\right)+\gamma\right\}
$$

Now consider two cases for $h$. If $h<h_{t}$ condition (69) becomes

$$
\operatorname{Tr}(H W)+\beta \nu(S)+\gamma \leq \mu(\beta+\gamma) .
$$

On the other hand if $h \geq h_{t}$ we have that $q_{\max }(h) \geq q_{\max }\left(h_{t}\right)$ by monotonicity of $q_{\max }$, so we may bound $\psi(h)=1-$ $q_{\max }(h) \leq 1-q_{\max }\left(h_{t}\right)$. Condition (69) becomes

$$
\operatorname{Tr}(H W)+\left(1-q_{\max }\left(h_{t}\right)\right)\{\beta \nu(S)+\gamma\} \leq \mu \gamma .
$$

We pick a $\gamma>$ to satisfy (71) with equality, that is

$$
\gamma=\frac{\left(1-q_{\max }\left(h_{t}\right)\right) \nu(S) \beta+\operatorname{Tr}(H W)}{\mu-\left(1-q_{\max }\left(h_{t}\right)\right)}
$$

where the denominator is positive by the choice of $\mu$ in (53). We will show that condition (70) also holds by an appropriate choice for $\beta>0$.

Case $\|\varepsilon\|<L$. In this case $q \geq 0 \Rightarrow 1-q \leq 1$ and it is sufficient for (67) to show that

$$
\begin{aligned}
& \sup _{\|\varepsilon\|<L} \varepsilon^{T}(1-\bar{q}) A^{T} H A \varepsilon+\operatorname{Tr}(H W)+\beta \nu(S)+\gamma \\
& \leq \mu(\beta+\gamma)
\end{aligned}
$$


holds, where on the right hand side we lower bounded the quadratic term of $W$ by 0 . This is of the general form

$$
C_{2}+\beta \nu(S)+\gamma \leq \mu(\beta+\gamma)
$$

for some constant $C_{2}$, and recall that the left over condition (70) is of the same form. Plugging the chosen $\gamma$ by (72) in (74) leads to a condition of the form

$$
C_{3} \leq\left(1-\frac{q_{\max }\left(h_{t}\right) \nu(S)}{\mu-\left(1-q_{\max }\left(h_{t}\right)\right)}\right) \mu \beta,
$$

for some constant $C_{3}$. We want to be able select $\beta>0$ that satisfies (75) for any value of the constant $C_{3}$. Hence we require the coefficient of $\beta$ to be strictly positive. This turns out to be equivalent to $\mu>1-q_{\max }\left(h_{t}\right)+q_{\max }\left(h_{t}\right) \nu(S)$, which corresponds to our choice of $\mu$ in (53). Therefore we conclude that part (iii) of the lemma holds as well and this completed the proof.

Returning to the proof of Proposition 2, combining (48) with condition (50) of the above Lemma we have that $\mathbb{E}^{\pi} e_{k}^{T} e_{k} \leq$ $K \mathbb{E}^{\pi} W\left(\varepsilon_{k}, h_{k}\right)$, so it suffices for (20) to show that a uniform bound on the expected value of $W\left(\varepsilon_{k}, h_{k}\right)$ exists.

By condition (ii) of the above lemma for $\left(B_{1}, B_{2}\right)=$ $\left(\mathbb{R}^{n}, \mathbb{R}_{+}\right)$we have that $\phi(\varepsilon, h, q) \leq 1 / \nu\left(\mathbb{R}^{n}, \mathbb{R}_{+}\right)$. Plugging this in (iii) leads to

$$
\mathbb{E}\left[W\left(\varepsilon^{+}, h^{+}\right) \mid \varepsilon, h, q\right] \leq \mu W(\varepsilon, h)+\nu(W) / \nu\left(\mathbb{R}^{n}, \mathbb{R}_{+}\right)
$$

Iterated applications of this inequality across some policy $\pi \in$ $\Pi$ yields

$$
\mathbb{E}^{\pi} W\left(\varepsilon_{k}, h_{k}\right) \leq \mu^{k} \mathbb{E} W\left(\varepsilon_{0}, h_{0}\right)+\frac{\nu(W)}{(1-\mu) \nu\left(\mathbb{R}^{n}, \mathbb{R}_{+}\right)}
$$

Thus since $\mu<1$ a uniform bound on $\mathbb{E}^{\pi} W\left(\varepsilon_{k}, h_{k}\right)$ exists and this completes the proof.

\section{B. Proof of Theorem 1}

First note that since $\Sigma_{k}:=\mathbb{E}^{\pi}\left[e_{k} e_{k}^{T} \mid G_{k}\right]$ we have that

$$
\mathbb{E}^{\pi}\left[\operatorname{Tr}\left(\Sigma_{k}\right)\right]=\mathbb{E}^{\pi} e_{k}^{T} e_{k}
$$

Then under Assumption 1, Proposition 2 states that for any $\pi \in \Pi$ condition (20) holds and guarantees that both quantities in (78) are bounded uniformly over $k$.

To establish the optimality of the proposed control law we use the fact that the Bellman-like equation

$$
\begin{aligned}
& V\left(G_{k}\right)+\operatorname{Tr}(P W)+\operatorname{Tr}\left(\tilde{P} \Sigma_{k}\right)= \\
& \min _{u_{k}} \mathbb{E}^{\pi}\left[x_{k}^{T} Q x_{k}+u_{k}^{T} R u_{k}+V\left(G_{k+1}\right) \mid G_{k}, u_{k}\right],
\end{aligned}
$$

is satisfied for the function

$$
V\left(G_{k}\right)=\mathbb{E}^{\pi}\left[x_{k}^{T} P x_{k} \mid G_{k}\right],
$$

with $V\left(G_{0}\right)=x_{0}^{T} P x_{0}$, where $P$ is the solution to the standard algebraic Riccati equation and $\tilde{P}$ is given by (23). The existence of $P$ is guaranteed by the stabilizability of $(A, B)$ and detectability of $\left(A, Q^{1 / 2}\right)$.
Indeed observe that we can use the tower property to rewrite the term on the right hand side of (79) as

$$
\begin{aligned}
& \mathbb{E}^{\pi}\left[V\left(G_{k+1}\right) \mid G_{k}, u_{k}\right]=\mathbb{E}^{\pi}\left[x_{k+1}^{T} P x_{k+1} \mid G_{k}, u_{k}\right] \\
& =\mathbb{E}^{\pi}\left[\left(A x_{k}+B u_{k}\right)^{T} P\left(A x_{k}+B u_{k}\right) \mid G_{k}, u_{k}\right]+\operatorname{Tr}(P W),
\end{aligned}
$$

where the last equality follows by substituting $x_{k+1}$ from the system equation (1). The quadratic minimization over $u_{k}$ at the right hand side of (79) takes the standard form appearing in LQR problems with partial state information - see e.g. [29, Vol. II, Section 5.2]. The argument of the minimization in (79) is given by the control law (24). Straightforward substitutions show that the optimal value of the minimization equals the left hand side of (79).

The equation (79) can be used to show that the optimal control policy is (24). First iterate (79) for $k=0, \ldots, N-1$ across some control policy $\theta \in \Theta$ to get

$$
\begin{aligned}
& V\left(G_{0}\right)+N \operatorname{Tr}(P W)+\mathbb{E}^{\pi} \sum_{k=0}^{N-1} \operatorname{Tr}\left(\tilde{P} \Sigma_{k}\right) \\
& \leq J_{\mathrm{LQR}}^{N}(\pi, \theta)+\mathbb{E}^{\pi, \theta} V\left(G_{N}\right)
\end{aligned}
$$

Dividing (82) by $N$ and taking the limit as $N \rightarrow \infty$, the term on the left hand side tends to

$$
\begin{aligned}
& \limsup _{N \rightarrow \infty} 1 / N\left[x_{0}^{T} P x_{0}+N \operatorname{Tr}(P W)+\mathbb{E}^{\pi} \sum_{k=0}^{N-1} \operatorname{Tr}\left(\tilde{P} \Sigma_{k}\right)\right] \\
& =\operatorname{Tr}(P W)+\underset{N \rightarrow \infty}{\limsup } 1 / N \mathbb{E}^{\pi} \sum_{k=0}^{N-1} e_{k}^{T} \tilde{P} e_{k}
\end{aligned}
$$

where we used (78) to convert $\Sigma_{k}$ to $e_{k}$.

Then consider the term on the right hand side of (82). Any control policy $\theta \in \Theta$ satisfying (21) also satisfies

$$
\lim _{N \rightarrow \infty} \frac{1}{N} \mathbb{E}^{\pi, \theta} V\left(G_{N}\right)=\lim _{N \rightarrow \infty} \frac{1}{N} \mathbb{E}^{\pi, \theta} x_{N}^{T} P x_{N}=0
$$

by the form of $V$ given in (80). Thus taking the limit as $N \rightarrow$ $\infty$, by (84) the term on the right hand side of (82) tends to the average LQR cost. The inequality in (82) then shows that the average LQR cost of $\theta$ is larger or equal to the limit of the left hand side which was given in (83). The result (22) of the theorem follows by including the power cost that depends only on the communication policy $\pi$ as suggested by (19).

The final step of the proof is to show that the control policy $\theta^{*}$ defined by (24) gives exactly the LQR cost given in (83). This policy satisfies (79) with equality, so (82) also holds with equality for $\theta^{*}$. Dividing by $N$ and taking the limit as before would prove the desired result if condition (84) also holds for $\theta^{*}$. We next verify that this is the case.

Indeed use $u_{k}^{*}=K \hat{x}_{k}$ and $x_{k}=\hat{x}_{k}+e_{k}$ to rewrite the closed loop system equation (1) under $\theta^{*}$ as

$$
x_{k+1}=(A+B K) \hat{x}_{k}+A e_{k}+w_{k} .
$$

Then denoting $\rho:=\lambda_{\max }(A+B K)$ which is stable, $\rho<1$, we can upper bound (81) under $\theta^{*}$ by

$$
\begin{aligned}
& \mathbb{E}^{\pi, \theta^{*}}\left[V\left(G_{k+1}\right) \mid G_{k}\right] \\
& \leq \rho^{2} \hat{x}_{k}^{T} P \hat{x}_{k}+\operatorname{Tr}\left(A^{T} P A \Sigma_{k}\right)+\operatorname{Tr}(P W) \\
& =\rho^{2} V\left(G_{k}\right)+\operatorname{Tr}\left(\left(A^{T} P A-\rho^{2} P\right) \Sigma_{k}\right)+\operatorname{Tr}(P W)
\end{aligned}
$$


where in the last equality we used the fact that $\hat{x}_{k} \hat{x}_{k}^{T}=$ $\mathbb{E}^{\pi}\left[x_{k} x_{k}^{T} \mid G_{k}\right]-\Sigma_{k}$. Taking expectation on both sides of (86) we have that

$$
\begin{aligned}
& \mathbb{E}^{\pi, \theta^{*}} V\left(G_{k+1}\right) \leq \rho^{2} \mathbb{E}^{\pi, \theta^{*}} V\left(G_{k}\right) \\
& \quad+\operatorname{Tr}\left(\left(A^{T} P A-\rho^{2} P\right) \mathbb{E}^{\pi, \theta^{*}} \Sigma_{k}\right)+\operatorname{Tr}(P W)
\end{aligned}
$$

But (78) and (20) imply that $\mathbb{E}^{\pi, \theta^{*}} \Sigma_{k}$ is uniformly bounded over $k$ so the term on the second line of (87) is bounded by some constant $\delta<\infty$. Iterating the above inequality (87) across $\theta^{*}$ up to $k=N$ yields

$$
\mathbb{E}^{\pi, \theta^{*}}\left[x_{N}^{T} P x_{N}\right] \leq \rho^{2 N} x_{0}^{T} P x_{0}+\delta /\left(1-\rho^{2}\right)
$$

which guarantees the limit (84) since $\rho<1$.

\section{Proof of Theorem 2}

The proof of the theorem is a direct application of the theorems contained in [31]. For these we need to show that [31, Assumptions 3.1, 3.2, 3.4] hold in our case. In particular [31, Assumption 3.1] requires that the cost per stage is bounded $|c(\varepsilon, h, q)| \leq K W(\varepsilon, h)$ by a positive measurable function $W$. This is a consequence of (50) of Lemma 1 , since

$$
|c(\varepsilon, h, q)| \leq(1-q) \lambda_{\max }(\tilde{P}) \varepsilon^{T} \varepsilon+\lambda p_{\max }
$$

which is of the same form as (50). Also [31, Assumption 3.2] requires exactly the conditions given in (i)-(iv) of Lemma 1. Finally [31, Assumption 3.4] requires the following conditions for the functions $W$ and $\phi$ satisfying Lemma 1.

Assumption 3. For every $\varepsilon \in \mathbb{R}^{n}, h \in \mathbb{R}_{+}$

(i) $Q(\varepsilon, h)$ is compact,

(ii) $c(\varepsilon, h, q)$ is lower semi-continuous in $q \in Q(\varepsilon, h)$,

(iii) $\mathbb{P}\left(\varepsilon^{+}, h^{+} \mid \varepsilon, h, q\right)$ is strongly continuous ${ }^{1}$ in $q \in Q(\varepsilon, h)$,

(iv) the mapping $q \rightarrow \mathbb{E}\left[W\left(\varepsilon^{+}, h^{+}\right) \mid \varepsilon, h, q\right]$ is continuous,

(v) $\phi(\varepsilon, h, q)$ is continuous in $q \in Q(\varepsilon, h)$.

Part (i) is trivial, and (ii) is a consequence of the continuity of $p(h, q)$ by Assumption 2. Strong continuity in (iii) is guaranteed by the fact that the transition kernel given in (29) has a probability density function. Part (iv) holds because the transition (29) is linear in $q$, and (v) is trivial.

Having established [31, Assumptions 3.1, 3.2, 3.4], then [31, Theorems 3.5, 3.6] state that in our case the infimum $J_{\text {COMM }}^{*}$ in (27) exists, there exists a function $V(\varepsilon, h)$ that satisfies (30), and the optimal policy is the minimizer of the right hand side of (30) as given in (31).

Finally note that (30) holds if we add any constant to $V(\varepsilon, h)$, so without loss of generality we may take $V(0, \hat{h})=$ 0 for some $\hat{h}$. Then for $\varepsilon=0, h=\hat{h}$ (30) gives

$$
\begin{aligned}
& V(0, \hat{h})=0=\min _{q \in Q(0, \hat{h})}\left\{c(0, \hat{h}, q)-J_{\text {COMM }}^{*}\right. \\
&\left.+\mathbb{E}\left[V\left(\varepsilon^{+}, h^{+}\right) \mid 0, \hat{h}, q\right]\right\} .
\end{aligned}
$$

${ }^{1}$ i.e. for every bounded measurable function $\Psi$ on $\mathbb{R}^{n} \times \mathbb{R}_{+}$, the mapping $q \mapsto \mathbb{E}\left[\Psi\left(\varepsilon^{+}, h^{+}\right) \mid \varepsilon, h, q\right]$ is continuous
Note that by (29), $\mathbb{P}\left(\varepsilon^{+}, h^{+} \mid 0, \hat{h}, q\right)=\mathcal{N}_{0, W}\left(\varepsilon^{+}\right) m_{H}\left(h^{+}\right)$, and also $c(0, \hat{h}, q)=\lambda p(\hat{h}, q)$ by (28), so (90) becomes

$$
0=\min _{q \in Q(0, \hat{h})}\left\{\lambda p(\hat{h}, q)-J_{\text {COMM }}^{*}+\mathbb{E}_{w, h} V(w, h)\right\} .
$$

The minimizer is $q=0$, giving the optimal value $J_{\text {COMM }}^{*}=$ $\mathbb{E}_{w, h} V(w, h)$ provided in the statement of the theorem.

\section{Proof of Theorem 3}

For any channel-dependent policy $q(h)$ with expected success $\bar{q}$ the error $e_{k}$ in (17) is a Markov jump linear system, with mean $\mathbb{E}\left(e_{k}\right)=0$ for all $k$ and covariance

$$
\mathbb{E}\left(e_{k} e_{k}^{T}\right)=(1-\bar{q})\left(A \mathbb{E}\left(e_{k-1} e_{k-1}^{T}\right) A^{T}+W\right) .
$$

Since $\bar{q}>q_{\text {crit }}$ the covariance reaches a steady state matrix $E$ that satisfies the Lyapunov equation (42), and $e_{k}$ is stable in the bounded covariance sense. The cost of such a policy is then given by (41).

The corresponding relative value function $V^{q(\cdot)}(\varepsilon, h)$ satisfies the steady state condition [c.f. (30)]

$$
\begin{aligned}
& V^{q(\cdot)}(\varepsilon, h)+J^{q(\cdot)} \\
& =c(\varepsilon, h, q(h))+\mathbb{E}\left[V^{q(\cdot)}\left(\varepsilon^{+}, h^{+}\right) \mid \varepsilon, h, q(h)\right],
\end{aligned}
$$

where $c(\varepsilon, h, q)$ is given by (28). We need to show that $V^{q(\cdot)}(\varepsilon, h)$ in (43) satisfies condition (93). First integrate (43) with respect to (29) to get

$$
\begin{aligned}
& \mathbb{E}\left[V^{q(\cdot)}\left(\varepsilon^{+}, h^{+}\right) \mid \varepsilon, h, q(h)\right]=(1-q(h)) \varepsilon^{T} A^{T} H A \varepsilon \\
& +\operatorname{Tr}(H W)+\lambda \mathbb{E}_{h^{+}} p\left(h^{+}, q\left(h^{+}\right)\right) .
\end{aligned}
$$

Using (94), the total cost $J^{q(\cdot)}$ in (41), and the cost per stage $c(\varepsilon, h, q)$ in (28), we conclude that $V^{q(\cdot)}(\varepsilon, h)$ in (43) satisfies condition (93) if the following equation holds

$$
\begin{aligned}
& \frac{1-q(h)}{1-\bar{q}} \varepsilon^{T} H \varepsilon+\lambda p(h, q(h))+\operatorname{Tr}(\tilde{P} E)+\lambda \mathbb{E}_{h} p(h, q(h)) \\
& =(1-q(h)) \varepsilon^{T} \tilde{P} \varepsilon+\lambda p(h, q(h))+(1-q(h)) \varepsilon^{T} A^{T} H A \varepsilon \\
& +\operatorname{Tr}(H W)+\lambda \mathbb{E}_{h^{+}} p\left(h^{+}, q\left(h^{+}\right)\right) .
\end{aligned}
$$

Substituting $H$ by (44) on the left hand side and canceling terms, the above condition becomes equivalent to $\operatorname{Tr}(\tilde{P} E)=$ $\operatorname{Tr}(H W)$. This is easily verified if we substitute the explicit expressions for the solutions $E, H$ of the discrete-time Lyapunov equations (42), (44) given by

$$
\begin{aligned}
& E=\sum_{k=0}^{\infty}(1-\bar{q})^{k+1} A^{k} W\left(A^{T}\right)^{k}, \\
& H=\sum_{k=0}^{\infty}(1-\bar{q})^{k+1}\left(A^{T}\right)^{k} \tilde{P} A^{k},
\end{aligned}
$$

respectively. Existence and uniqueness of solutions to the Lyapunov equations (42), (44) follows by assumption $\bar{q}>q_{\text {crit }}$. 


\section{REFERENCES}

[1] K. Gatsis, A. Ribeiro, and G. J. Pappas, "Optimal power management in wireless control systems," in Proc. of the 2013 American Control Conference (ACC 2013), pp. 1562-1569, 2013.

[2] J. Hespanha, P. Naghshtabrizi, and Y. Xu, "A survey of recent results in networked control systems," Proceedings of the IEEE, vol. 95, no. 1, pp. 138-162, 2007.

[3] L. Schenato, B. Sinopoli, M. Franceschetti, K. Poolla, and S. Sastry, "Foundations of control and estimation over lossy networks," Proceedings of the IEEE, vol. 95, no. 1, pp. 163-187, 2007.

[4] V. Gupta, B. Hassibi, and R. Murray, "Optimal LQG control across packet-dropping links," Systems \& Control Letters, vol. 56, no. 6, pp. 439-446, 2007.

[5] O. C. Imer, S. Yüksel, and T. Başar, "Optimal control of LTI systems over unreliable communication links," Automatica, vol. 42, no. 9, pp. 1429-1439, 2006.

[6] N. Elia, "Remote stabilization over fading channels," Systems \& Control Letters, vol. 54, no. 3, pp. 237-249, 2005.

[7] J. H. Braslavsky, R. H. Middleton, and J. S. Freudenberg, "Feedback stabilization over signal-to-noise ratio constrained channels," IEEE Transactions on Automatic Control, vol. 52, no. 8, pp. 1391-1403, 2007.

[8] S. Tatikonda and S. Mitter, "Control under communication constraints," IEEE Transactions on Automatic Control, vol. 49, no. 7, pp. 1056-1068, 2004.

[9] G. Nair, F. Fagnani, S. Zampieri, and R. Evans, "Feedback control under data rate constraints: An overview," Proceedings of the IEEE, vol. 95, no. 1, pp. 108-137, 2007.

[10] K. J. Astrom and B. M. Bernhardsson, "Comparison of Riemann and Lebesgue sampling for first order stochastic systems," in Proc. of the 41st IEEE Conference on Decision and Control, vol. 2, pp. 2011-2016, 2002.

[11] M. Rabi, G. V. Moustakides, and J. S. Baras, "Multiple sampling for estimation on a finite horizon," in Proc. of the 45th IEEE Conference on Decision and Control, pp. 1351-1357, 2006.

[12] P. Tabuada, "Event-triggered real-time scheduling of stabilizing control tasks," IEEE Transactions on Automatic Control, vol. 52, no. 9, pp. 1680-1685, 2007.

[13] M. Rabi, K. Johansson, and M. Johansson, "Optimal stopping for eventtriggered sensing and actuation," in Proc. of the 47th IEEE Conference on Decision and Control., pp. 3607-3612, 2008.

[14] A. Anta and P. Tabuada, "To sample or not to sample: Self-triggered control for nonlinear systems," IEEE Transactions on Automatic Control, vol. 55, no. 9, pp. 2030-2042, 2010.

[15] Y. Xu and J. Hespanha, "Optimal communication logics in networked control systems," in Proc. of the 43rd IEEE Conference on Decision and Control, vol. 4, pp. 3527-3532, 2004.

[16] A. Mesquita, J. Hespanha, and G. Nair, "Redundant data transmission in control/estimation over lossy networks," Automatica, vol. 48, pp. 10201027,2012

[17] R. Cogill, S. Lall, and J. Hespanha, "A constant factor approximation algorithm for event-based sampling," in Proc. of the 2007 American Control Conference, pp. 305-311, 2007.

[18] L. Li and M. Lemmon, "Performance and average sampling period of sub-optimal triggering event in event triggered state estimation," in Proc. of the 50th IEEE Conference on Decision and Control and European Control Conference (CDC-ECC 2011), pp. 1656-1661, 2011.

[19] A. Molin and S. Hirche, "On LQG joint optimal scheduling and control under communication constraints," in Proc. of the 48th IEEE Conference on Decision and Control, pp. 5832-5838, 2009.

[20] C. Ramesh, H. Sandberg, and K. H. Johansson, "Design of statebased schedulers for a network of control loops," IEEE Transactions on Automatic Control,, vol. 58, no. 8, pp. 1962-1975, 2013.

[21] L. Bao, M. Skoglund, and K. H. Johansson, "Iterative encoder-controller design for feedback control over noisy channels," IEEE Transactions on Automatic Control, vol. 56, no. 2, pp. 265-278, 2011.

[22] A. Goldsmith, Wireless communications. Cambr. Univ. Press, 2005.

[23] D. E. Quevedo, A. Ahlén, A. S. Leong, and S. Dey, "On Kalman filtering over fading wireless channels with controlled transmission powers," Automatica, vol. 48, no. 7, pp. 1306-1316, 2012.

[24] A. S. Leong and S. Dey, "Power allocation for error covariance minimization in Kalman filtering over packet dropping links," in Proc. of the 51st IEEE Conference on Decision and Control, pp. 3335-3340, 2012.

[25] A. R. Mesquita, Exploiting Stochasticity in Multi-agent Systems. PhD thesis, University of California, 2010.
[26] J. Zhang, K. Tan, J. Zhao, H. Wu, and Y. Zhang, "A practical SNRguided rate adaptation," in INFOCOM 2008. The 27th Conference on Computer Communications., pp. 2083-2091, 2008.

[27] N. Ploplys, P. Kawka, and A. Alleyne, "Closed-loop control over wireless networks," IEEE Control Systems Magazine, vol. 24, no. 3, pp. 58-71, 2004.

[28] T. K. Moon, Error correction coding: mathematical methods and algorithms. Wiley, 2005.

[29] D. P. Bertsekas, Dynamic Programming and Optimal Control. Athena Scientific, 2005.

[30] O. Hernández-Lerma and J. Lasserre, Discrete-time Markov control processes: basic optimality criteria. Springer New York, 1996.

[31] O. Vega-Amaya, "The average cost optimality equation: a fixed point approach," Bol. Soc. Mat. Mexicana, vol. 9, no. 1, pp. 185-195, 2003.

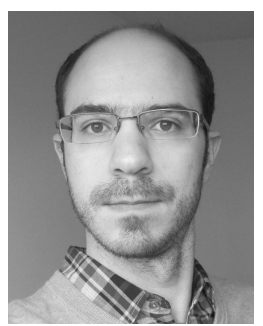

Konstantinos Gatsis (S'10) received the Diploma degree in electrical and computer engineering from the University of Patras, Patras, Greece in 2010. Currently, he is working toward the Ph.D. degree in the Department of Electrical and Systems Engineering, University of Pennsylvania, Philadelphia. His research interests include cyber-physical systems, networked control systems, as well as resource optimization problems arising in them.

Mr. Gatsis received the Student Best Paper Award at the 2013 American Control Conference.

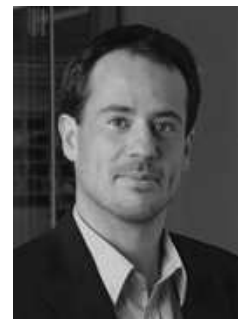

Alejandro Ribeiro (S'02-M'07) received the B.Sc. degree in electrical engineering from the Universidad de la Republica Oriental del Uruguay, Montevideo, in 1998 and the M.Sc. and Ph.D. degree in electrical engineering from the Department of Electrical and Computer Engineering, the University of Minnesota, Minneapolis in 2005 and 2007.

From 1998 to 2003, he was a member of the technical staff at Bellsouth Montevideo. After his M.Sc. and $\mathrm{Ph} . \mathrm{D}$ studies, in 2008 he joined the University of Pennsylvania (Penn), Philadelphia, where he is currently an Assistant Professor at the Department of Electrical and Systems Engineering. His research interests are in the applications of statistical signal processing to the study of networks and networked phenomena. His current research focuses on wireless networks, network optimization, learning in networks, networked control, robot teams, and structured representations of networked data structures.

Dr. Ribeiro received the 2012 S. Reid Warren, Jr. Award presented by Penn's undergraduate student body for outstanding teaching and the NSF CAREER Award in 2010. He is also a Fulbright scholar and the recipient of student paper awards at the 2013 American Control Conference (as adviser), as well as the 2005 and 2006 International Conferences on Acoustics, Speech and Signal Processing.

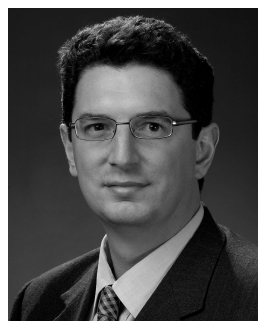

George J. Pappas (S'90-M'91-SM'04-F'09) received the Ph.D. degree in electrical engineering and computer sciences from the University of California, Berkeley (where he received the Eliahu Jury Award for Excellence in Systems Research), in 1998.

$\mathrm{He}$ is currently the Joseph Moore Professor of Electrical and Systems Engineering at the University of Pennsylvania, Philadelphia. He is a member of the General Robotics, Automation, Sensing and Perception (GRASP) Laboratory and serves as the Chair of the Department of Electrical and Systems Engineering. His current research interests include hybrid and embedded systems, hierarchical control systems, distributed control systems, nonlinear control systems, with applications to robotics, unmanned aerial vehicles, biomolecular networks, and green buildings.

Dr. Pappas has received numerous awards, including the National Science Foundation (NSF) CAREER Award in 2002, the NSF Presidential Early Career Award for Scientists and Engineers in 2002, the 2009 George S. Axelby Outstanding Paper Award, and the 2010 Antonio Ruberti Outstanding Young Researcher Prize. 